Article

\title{
The Changes in Bioactive Compounds and Antioxidant Activity of Chia (Salvia hispanica L.) Herb under Storage and Different Drying Conditions: A Comparison with Other Species of Sage
}

\author{
Kinga Dziadek ${ }^{1, *(\mathbb{D})}$, Aneta Kopeć ${ }^{1}\left(\mathbb{D}\right.$, Michał Dziadek ${ }^{2,3}{ }^{\mathbb{D}}$, Urszula Sadowska ${ }^{4}$ and \\ Katarzyna Cholewa-Kowalska ${ }^{3}$ (D)
}

1 Department of Human Nutrition and Dietetics, Faculty of Food Technology, University of Agriculture in Krakow, 21 Mickiewicza Ave., 31-120 Krakow, Poland; aneta.kopec@urk.edu.pl

2 Department of Inorganic Chemistry, Faculty of Chemistry, Jagiellonian University, 2 Gronostajowa St., 30-387 Krakow, Poland; michal.dziadek@uj.edu.pl or dziadek@agh.edu.pl

3 Department of Glass Technology and Amorphous Coatings, Faculty of Materials Science and Ceramics, AGH University of Science and Technology, 30 Mickiewicza Ave., 30-059 Krakow, Poland; cholewa@agh.edu.pl

4 Institute of Machinery Exploitation, Ergonomics and Production Processes, Faculty of Production and Power Engineering, University of Agriculture in Krakow, 21 Mickiewicza Ave., 31-120 Krakow, Poland; urszula.sadowska@urk.edu.pl

* Correspondence: kinga.dziadek@urk.edu.pl; Tel.: +48-12-662-4717

check for updates

Citation: Dziadek, K.; Kopeć, A.; Dziadek, M.; Sadowska, U.; Cholewa-Kowalska, K. The Changes in Bioactive Compounds and Antioxidant Activity of Chia (Salvia hispanica L.) Herb under Storage and Different Drying Conditions: A Comparison with Other Species of Sage. Molecules 2022, 27, 1569. https://doi.org/10.3390/molecules 27051569

Academic Editors: Gabriele Rocchetti and Danijela Bursać Kovačević

Received: 1 February 2022

Accepted: 24 February 2022

Published: 26 February 2022

Publisher's Note: MDPI stays neutral with regard to jurisdictional claims in published maps and institutional affiliations.

Copyright: (C) 2022 by the authors. Licensee MDPI, Basel, Switzerland. This article is an open access article distributed under the terms and conditions of the Creative Commons Attribution (CC BY) license (https:// creativecommons.org/licenses/by/ $4.0 /)$.
Abstract: Studies on herb chia (Salvia hispanica L.) are very limited. Therefore, the aim of this study was to assess how different drying methods and periods of storage affect the bioactive properties of the herb Salvia hispanica and to compare it with other species of sage (Salvia officinalis L. and Salvia sclarea L.). In fresh herbs, directly after drying (freeze-drying, natural drying, and drying at 30, 40, and $\left.50{ }^{\circ} \mathrm{C}\right)$, and after storage $(3,6$, and 12 months), the following analyses were performed: content of total carotenoids and total polyphenols, polyphenol profile (including 25 compounds), and antioxidant activity. Additionally, the basic chemical compositions of the herbs were analyzed. To the best of our knowledge, the content of total carotenoids and the quantitative polyphenol profile in Salvia hispanica and Salvia sclarea were evaluated for the first time. The obtained results showed that the barely investigated herb Salvia hispanica is rich in polyphenolic compounds and shows high antioxidant activity. In all the tested species, rosmarinic acid was the most abundant polyphenolic compound. The use of different drying methods allowed us to determine that freeze-drying was the most effective for preserving polyphenols and carotenoids. Long-term storage up to 12 months resulted in a gradual reduction in antioxidant activity and in the content of polyphenols and carotenoids.

Keywords: sage; Salvia hispanica herb; chia herb; drying; freeze-drying; storage; polyphenols

\section{Introduction}

Herbs have been used for centuries as natural remedies to fight some diseases. With the development of synthetic drugs in the early 20th century, the use of herbs in medicine declined significantly. However, in recent years, there has been growing interest in folk medicine involving medical plants and herbs [1-3].

One group of beneficial medicinal plants is the genus Salvia, which belongs to the Lamiaceae family and includes approximately 900 species [4]. It is cultivated throughout the world for use in food industries as spices (to flavor meats such as pork, sausage, and poultry) as well as in cosmetics [5,6], newly formulated biomaterials [7-9], and active food packaging [10]. Studies have indicated that several species of sage (including Salvia officinalis L. and Salvia sclarea L.) show biological activities such as antioxidant, antibacterial, antifungal, anticancer, antimutagenic, anti-inflammatory, and antidepressant 
activities [5,6,11-16]. These beneficial effects result from numerous compounds belonging to different chemical groups, mainly polyphenolic compounds (phenolic acids and flavonoids), carotenoids, and essential oils [12,17].

Fresh herbs are treated using various preservation processes, to extend their shelf life while maintaining the highest quality. To protect their microbiological stability and biological activities, the level of water must be decreased below 10\% [18]. Drying is one of the most commonly used methods of preservation. It reduces microbial growth, and therefore allows for long-term storage. Furthermore, during the drying process some biochemical changes occur that can improve the quality. Moreover, freeze-drying is an increasingly used drying method in the food industry that preserves the properties of the fresh plant (including chemical composition and biological activities) to the greatest extent. Additionally, these treatments allow for a decrease in the cost of transport and storage by reducing the product weight [19-21]. Nowadays, innovative drying technologies such as the reaction engineering approach (REA), as well as microwave, infrared, ultrasonic, lowpressure superheated steam, and pulse combustion spray drying are under investigation. These methods are promising prospects for the food industry. They can enhance the quality of the product, as well as improve the drying efficiency, reducing energy consumption and environmental impact $[22,23]$.

Salvia hispanica L. (known as chia) is cultivated mainly for its seeds. The growing interest in chia in recent years has been due to its beneficial fatty acid profile. Chia seeds contain highly unsaturated fatty acids, mainly linoleic and $\alpha$-linolenic acid $[24,25]$. They are a source of protein, with a proper balance of the essential amino acids, especially methionine and cysteine [26]. Chia seeds also contain bioactive compounds, mainly dietary fiber but also polyphenolic compounds such as myricetin, quercetin, kaempferol, and chlorogenic acids $[25,27,28]$. However, there are limited studies on the herb of Salvia hispanica $[27,29]$.

The aim of this study was to assess how different drying methods and periods of storage affect the content of bioactive compounds (including the polyphenol profile) and antioxidant activity of the herb Salvia hispanica, as well as to compare it with other species of sage (Salvia officinalis and Salvia sclarea). To the best of our knowledge, the content of total carotenoids and the quantitative polyphenol profile in Salvia hispanica and Salvia sclarea were evaluated for the first time in this study.

\section{Materials and Methods}

\subsection{Plant Material}

The following species of sage were analyzed: Salvia hispanica (chia), Salvia officinalis, and Salvia sclarea. All species were grown in the same location (Bielsko-Biała, Silesia, Poland) and under the same conditions. No pesticides and no hydration were used during cultivation. The herb samples were randomly collected from plants. The samples were dried using the following methods: freeze-drying, natural drying, and drying at $30^{\circ} \mathrm{C}, 40{ }^{\circ} \mathrm{C}$, and $50{ }^{\circ} \mathrm{C}$. Lyophilization was conducted in a vacuum freeze dryer (Alpha 1-4 LSCplus, Martin Christ Gefriertrocknungsanlagen $\mathrm{GmbH}$, Osterode am Harz, Germany). Natural drying was performed in a dry and shaded room, while convection drying at temperatures of $30^{\circ} \mathrm{C}$, $40^{\circ} \mathrm{C}$, and $50^{\circ} \mathrm{C}$ was conducted in a laboratory dryer. The volume of the dryer working chamber was $100 \mathrm{dm}^{3}$. Natural drying and drying at elevated temperatures were carried out until a water content of $100 \mathrm{~mL} \cdot \mathrm{kg}^{-1}$ was achieved. Additionally, after drying, herbs were tightly packed and stored for 3,6 , and 12 months at room temperature, protected from sunlight. Fresh and dried herbs, before and after the different storage periods, were used to prepare methanolic extracts and to evaluate the total carotenoids content. Additionally, the freeze-dried herbs were used to determine the basic chemical composition.

\subsection{Basic Chemical Composition}

In the freeze-dried samples, the contents of total protein (procedure no. 950.36), crude fat (procedure no. 935.38), total dietary fiber (procedure no. 991.43) and ash (procedure no. 
930.05) were determined according to the AOAC [30] methods. The content of digestible carbohydrates was calculated using the following equation [31]:

digestible carbohydrates $=100-($ protein + crude fat + ash + dietary fiber $)$

\subsection{Determination of Total Carotenoids}

The content of total carotenoids was determined by extracting carotenoids from the samples using an acetone-hexane mixture (4:6 v/v) (Chempur, Piekary Śląskie, Poland), according to the Polish standard with some modifications [32]. Samples containing $1 \mathrm{~g}$ of fresh or $0.2 \mathrm{~g}$ of dried sage herbs were weighed into a porcelain mortar. Thereafter, approximately $0.3 \mathrm{~g}$ of sand was used to extract the dye using an acetone-hexane mixture. The extracts were poured into a test cylinder. Then, the extract volume was read. The extracts were shaken and left in a dark place for $30 \mathrm{~min}$. The absorbance was measured at $450 \mathrm{~nm}$ using a spectrophotometer (UV-1800, Rayleigh, Beijing Beifen-Ruili Analytical Instrument Co., Ltd., Beijing, China). Results were calculated based on the $\beta$-carotene (Sigma-Aldrich, St. Louis, MO, USA) calibration curve.

\subsection{Preparation of the Extracts}

The content of total polyphenols, the polyphenol profile, and the antioxidant activity were determined in methanolic extracts. To prepare the extracts, $0.3 \mathrm{~g}$ of fresh or $0.2 \mathrm{~g}$ of dried herbs, were used, with $60 \mathrm{~mL}$ of $0.1 \%$ formic acid in $70 \%$ methanol $(v / v)(\mathrm{POCH}$, Gliwice, Poland). The extracts were prepared by shaking for $2 \mathrm{~h}$ at room temperature (water bath shaker type 357, Elpan, Lubawa, Poland). Then the samples were filtered using filter paper. The filtrates were stored at a temperature of $-20^{\circ} \mathrm{C}$ for further analysis.

\subsection{Determination of Total Polyphenols}

The level of total polyphenols was estimated using Folin-Ciocalteu reagent (SigmaAldrich, Saint-Louis, MO, USA), as previously described [33]. Results were expressed as $\mathrm{mg}$ of gallic acid (GA) per $100 \mathrm{~g}$ DW (dry weight) of the sample.

\subsection{Determination of Polyphenol Profile}

High-performance liquid chromatography (HPLC) was used to evaluate the polyphenol profile. The analysis was conducted using a Prominence-i LC-2030C 3D Plus system (Shimadzu, Kyoto, Japan) equipped with a diode array detector (DAD). The separation was performed on a Luna Omega $5 \mu \mathrm{m}$ Polar C18, $100 \mathrm{~A}, 250 \times 10 \mathrm{~mm}$ column (Phenomenex, CA, USA) at $40{ }^{\circ} \mathrm{C}$. The mobile phase was a mixture of two eluents: $\mathrm{A}-0.1 \%$ formic acid in water $(v / v)$ and $\mathrm{B}-0.1 \%$ formic acid in methanol $(v / v)$. The flow rate of the mobile phase was $1.2 \mathrm{~mL} \mathrm{~min}-1$. The analysis was carried out with the following gradient conditions: from $20 \%$ to $40 \%$ B in $10 \mathrm{~min}, 40 \%$ B for $10 \mathrm{~min}$, from $40 \%$ to $50 \%$ B in $10 \mathrm{~min}$, from $50 \%$ to $60 \% \mathrm{~B}$ in $5 \mathrm{~min}, 60 \% \mathrm{~B}$ for $5 \mathrm{~min}$, from 60 to $70 \% \mathrm{~B}$ in $5 \mathrm{~min}$, from $70 \%$ to $90 \% \mathrm{~B}$ in $5 \mathrm{~min}$, $90 \%$ B for $5 \mathrm{~min}$, from $90 \%$ to $20 \%$ B (the initial condition) in $1 \mathrm{~min}$, and $20 \%$ B for $4 \mathrm{~min}$, resulting in a total run time of $60 \mathrm{~min}$. The injection volume was $20 \mu \mathrm{L}$.

The quantification of individual polyphenols in methanolic extracts was performed by establishing calibration curves using the standards. The calibration curves for the standards were linear with $R^{2}>0.995$.

The detection of 4-hydroxybenzoic acid, myricetin, quercetin, luteolin, and isorhamnetin was performed at $254 \mathrm{~nm}$, rutin at $256 \mathrm{~nm}$, vanillic acid at $260 \mathrm{~nm}$, kaempferol at $264 \mathrm{~nm}$, apigenin and acacetin at $267 \mathrm{~nm}$, gallic acid at $271 \mathrm{~nm}$, hispidulin at $273 \mathrm{~nm}$, syringic acid at $274 \mathrm{~nm}$, catechin and epicatechin at $278 \mathrm{~nm}$, naringin and carnosol at $283 \mathrm{~nm}$, hesperidin and carnosic acid at $284 \mathrm{~nm}, \mathrm{p}$-coumaric acid at $310 \mathrm{~nm}$, caffeic acid, ferulic acid, and sinapinic acid at $323 \mathrm{~nm}$, chlorogenic acid at $326 \mathrm{~nm}$, and rosmarinic acid at $329 \mathrm{~nm}$. The data were integrated and analyzed using the LabSolutions software (Shimadzu, Kyoto, Japan). 


\subsection{Determination of Antioxidant Activity}

The antioxidant activity was determined using the ABTS method (2,2'-azino-bis(3ethylbenzthiazoline-6-sulfonic acid)), as previously reported [34]. The obtained results were expressed as micromoles of Trolox equivalent per gram (DW) of the sample.

\subsection{Statistical Analysis}

Statistical analysis of the obtained data was performed using the program Statistica version 13.1, Dell Inc., Tulsa, OK, USA, 2016. The results were analyzed using one-way analysis of variance (ANOVA). Significant differences between mean values $(n=3)$ were compared using Duncan's test.

\section{Results}

\subsection{Basic Chemical Composition}

Salvia sclarea was characterized by significantly the highest level of ash $(18.39 \mathrm{~g} \cdot 100$ $\mathrm{g}^{-1} \mathrm{DW}$ ) and protein (14.49 $\left.\mathrm{g} \cdot 100 \mathrm{~g}^{-1} \mathrm{DW}\right)$ (Table 1). The highest amount of crude fat was determined in Salvia officinalis ( $4.06 \mathrm{~g} \cdot 100 \mathrm{~g}^{-1} \mathrm{DW}$ ), while the lowest was found in Salvia hispanica $\left(1.67 \mathrm{~g} \cdot 100 \mathrm{~g}^{-1} \mathrm{DW}\right)$ herb. The highest content of dietary fiber $\left(52.98 \mathrm{~g} \cdot 100 \mathrm{~g}^{-1}\right.$ DW) and, at the same time, the lowest level of digestible carbohydrates $\left(24.82 \mathrm{~g} \cdot 100 \mathrm{~g}^{-1}\right.$ DW) were measured in Salvia officinalis, in comparison to the other species.

Table 1. Basic chemical composition of individual species of sage.

\begin{tabular}{cccccc}
\hline Species & $\begin{array}{c}\text { Ash } \\
{\left[\mathbf{g} \cdot \mathbf{1 0 0} \mathbf{~ g}^{-\mathbf{1}} \mathbf{D W}\right]}\end{array}$ & $\begin{array}{c}\text { Protein } \\
{\left[\mathbf{g} \cdot \mathbf{1 0 0} \mathbf{~ g}^{-\mathbf{1}} \mathbf{D W}\right]}\end{array}$ & $\begin{array}{c}\text { Crude Fat } \\
{\left[\mathbf{g} \cdot \mathbf{1 0 0} \mathbf{~ g}^{-\mathbf{1}} \mathbf{~ D W}\right]}\end{array}$ & $\begin{array}{c}\text { Digestible } \\
\text { Carbohydrates } \\
{\left[\mathbf{g} \cdot \mathbf{1 0 0} \mathbf{g}^{-\mathbf{1}} \mathbf{~} \mathbf{D W}\right]}\end{array}$ & $\begin{array}{c}\text { Dietary Fiber } \\
{\left[\mathbf{g} \cdot \mathbf{1 0 0} \mathbf{g}^{-\mathbf{1}} \mathbf{D W}\right]}\end{array}$ \\
\hline Salvia hispanica & $11.73 \pm 0.26^{\mathrm{b}}$ & $9.43 \pm 0.28^{\mathrm{a}}$ & $1.67 \pm 0.04^{\mathrm{a}}$ & $30.26 \pm 1.21^{\mathrm{b}}$ & $46.90 \pm 1.40^{\mathrm{b}}$ \\
Salvia officinalis & $8.76 \pm 0.25^{\mathrm{a}}$ & $9.38 \pm 0.06^{\mathrm{a}}$ & $4.06 \pm 0.06^{\mathrm{c}}$ & $24.82 \pm 2.41^{\mathrm{a}}$ & $52.98 \pm 2.10^{\mathrm{c}}$ \\
Salvia sclarea & $18.39 \pm 0.24^{\mathrm{c}}$ & $14.49 \pm 0.32^{\mathrm{b}}$ & $1.99 \pm 0.11^{\mathrm{b}}$ & $28.17 \pm 1.36^{\mathrm{b}}$ & $36.96 \pm 1.20^{\mathrm{a}}$ \\
\hline
\end{tabular}

Results are expressed as mean $\pm \mathrm{SD}(n=3)$. DW-dry weight. Mean values with different letters $(\mathrm{a}-\mathrm{c})$ within the individual columns are statistically different $(p<0.05)$.

\subsection{The Content of Total Carotenoids}

Among the fresh herbs, significantly the highest content of total carotenoids was found in Salvia sclarea (109.06 mg $100 \mathrm{~g}^{-1} \mathrm{DW}$ ) (Table 2). The drying methods and storage significantly affected the content of these compounds (Table 3). The freeze-dried herbs showed the highest content of carotenoids $\left(71.04 \mathrm{mg} \cdot 100 \mathrm{~g}^{-1} \mathrm{DW}\right.$ in Salvia sclarea, $64.87 \mathrm{mg} \cdot 100$ $\mathrm{g}^{-1} \mathrm{DW}$ in Salvia hispanica, and $57.98 \mathrm{mg} \cdot 100 \mathrm{~g}^{-1} \mathrm{DW}$ in Salvia officinalis), indicating that this method caused the smallest loss in comparison to other drying methods. On the other hand, samples dried at $30^{\circ} \mathrm{C}$ were characterized by the lowest level of these compounds (42.24 mg.100 g ${ }^{-1}$ DW in Salvia sclarea, $27.93 \mathrm{mg} \cdot 100 \mathrm{~g}^{-1} \mathrm{DW}$ in Salvia hispanica, and $23.60 \mathrm{mg} \cdot 100 \mathrm{~g}^{-1} \mathrm{DW}$ in Salvia officinalis), resulting from the highest losses. Storage for 3, 6, and 12 months caused an increasing loss in carotenoid content in all tested species of sage. The highest loss was observed after 12 months of storage. The loss of carotenoids in the studied samples during storage seemed not to depend on the drying method.

Table 2. The content of bioactive compounds and antioxidant activity in fresh sage of individual species.

\begin{tabular}{cccc}
\hline Species & $\begin{array}{c}\text { Total Carotenoids } \\
{\left[\mathbf{m g} \cdot \mathbf{1 0 0} \mathbf{~ g}^{-\mathbf{1}} \mathbf{D W}\right]}\end{array}$ & $\begin{array}{c}\text { Total Polyphenols } \\
{\left[\mathbf{g} \cdot \mathbf{1 0 0} \mathbf{g}^{-\mathbf{1}} \mathbf{D W}\right]}\end{array}$ & $\begin{array}{c}\text { Antioxidant Activity } \\
{\left[\boldsymbol{\mu \mathbf { m o l }} \text { Trolox} \cdot \mathbf{g}^{-\mathbf{1}} \mathbf{D W}\right]}\end{array}$ \\
\hline Salvia hispanica & $103.02 \pm 0.10^{\mathrm{b}}$ & $9.76 \pm 0.52^{\mathrm{b}}$ & $713.26 \pm 36.72^{\mathrm{b}}$ \\
Salvia officinalis & $63.48 \pm 0.19^{\mathrm{a}}$ & $9.47 \pm 0.15^{\mathrm{b}}$ & $651.48 \pm 30.87^{\mathrm{ab}}$ \\
Salvia sclarea & $109.06 \pm 1.05^{\mathrm{c}}$ & $4.95 \pm 0.30^{\mathrm{a}}$ & $568.49 \pm 42.99^{\mathrm{a}}$ \\
\hline
\end{tabular}

Results are expressed as mean $\pm \mathrm{SD}(n=3)$. DW-dry weight. Mean values with different letters $(\mathrm{a}-\mathrm{c})$ within the individual columns are statistically different $(p<0.05)$. 
Table 3. The content of total carotenoids in dried and stored sage of individual species.

\begin{tabular}{|c|c|c|c|c|c|c|c|c|c|}
\hline \multirow[b]{3}{*}{ Species } & \multirow[b]{3}{*}{$\begin{array}{l}\text { Drying } \\
\text { Method }\end{array}$} & \multicolumn{8}{|c|}{ Storage } \\
\hline & & \multicolumn{2}{|c|}{ Directly after Drying } & \multicolumn{2}{|c|}{ After 3 Months } & \multicolumn{2}{|c|}{ After 6 Months } & \multicolumn{2}{|c|}{ After 12 Months } \\
\hline & & $\begin{array}{c}\text { Total } \\
\text { Carotenoids } \\
{[\mathrm{mg} \cdot 100} \\
\left.\mathrm{g}^{-1} \mathrm{DW}\right]\end{array}$ & $\begin{array}{c}\text { Changes * } \\
{[\%]}\end{array}$ & $\begin{array}{c}\text { Total } \\
\text { Carotenoids } \\
{[\mathrm{mg} \cdot 100} \\
\left.\mathrm{g}^{-1} \mathrm{DW}\right]\end{array}$ & $\begin{array}{c}\text { Changes ** } \\
{[\%]}\end{array}$ & $\begin{array}{c}\text { Total } \\
\text { Carotenoids } \\
{[\mathrm{mg} \cdot 100} \\
\left.\mathrm{g}^{-1} \mathrm{DW}\right]\end{array}$ & $\begin{array}{c}\text { Changes ** } \\
{[\%]}\end{array}$ & $\begin{array}{c}\text { Total } \\
\text { Carotenoids } \\
{[\mathrm{mg} \cdot 100} \\
\left.\mathrm{g}^{-1} \mathrm{DW}\right]\end{array}$ & $\begin{array}{c}\text { Changes ** } \\
{[\%]}\end{array}$ \\
\hline \multirow{5}{*}{$\begin{array}{c}\text { Salvia } \\
\text { hispanica }\end{array}$} & $\begin{array}{l}\text { freeze- } \\
\text { drying }\end{array}$ & $\begin{array}{l}64.87 \pm \\
0.68\end{array}$ & -37.03 & $\begin{array}{l}54.90 \pm \\
1.07 \mathrm{~h}, \mathrm{C}\end{array}$ & -15.36 & $\begin{array}{l}41.38 \pm \\
0.26^{\mathrm{h}, \mathrm{B}}\end{array}$ & -36.21 & $\begin{array}{l}29.24 \pm \\
1.15^{\mathrm{h}, \mathrm{A}}\end{array}$ & -54.92 \\
\hline & $\begin{array}{l}\text { natural } \\
\text { drying }\end{array}$ & $\begin{array}{l}37.48 \pm \\
0.91^{\mathrm{f}, \mathrm{D}}\end{array}$ & -63.62 & $\begin{array}{l}24.76 \pm \\
0.57 \text { e, } \mathrm{C}\end{array}$ & -33.93 & $\begin{array}{l}19.63 \pm \\
0.30 \mathrm{~d}, \mathrm{~B}\end{array}$ & -47.63 & $\begin{array}{c}13.94 \pm \\
0.23^{\mathrm{cd}, \mathrm{A}}\end{array}$ & -62.81 \\
\hline & drying at & $\begin{array}{l}27.93 \pm \\
0.18^{\mathrm{b}}, \mathrm{C}\end{array}$ & -72.89 & $\begin{array}{l}16.43 \pm \\
0.37^{a, B}\end{array}$ & -41.16 & $\begin{array}{l}14.26 \pm \\
0.09 \mathrm{~b}, \mathrm{~A}\end{array}$ & -48.95 & $\begin{array}{c}14.10 \pm \\
0.09 \mathrm{de}, \mathrm{A}\end{array}$ & -49.50 \\
\hline & $\begin{array}{c}\text { drying at } \\
40^{\circ} \mathrm{C}\end{array}$ & $\begin{array}{l}29.41 \pm \\
0.08^{c,}, \mathrm{C}\end{array}$ & -71.46 & $\begin{array}{l}21.04 \pm \\
0.07^{c, B}\end{array}$ & -28.45 & $\begin{array}{l}21.10 \pm \\
0.10^{\mathrm{e}, \mathrm{B}}\end{array}$ & -28.25 & $\begin{array}{l}20.22 \pm \\
0.09 \mathrm{~g}, \mathrm{~A}\end{array}$ & -31.25 \\
\hline & $\begin{array}{c}\text { drying at } \\
50^{\circ} \mathrm{C}\end{array}$ & $\begin{array}{l}33.79 \pm \\
0.27^{\mathrm{e}}, \mathrm{D}\end{array}$ & -67.20 & $\begin{array}{l}23.09 \pm \\
0.22 \mathrm{~d}, \mathrm{C}\end{array}$ & -31.67 & $\begin{array}{l}18.73 \pm \\
0.87 \mathrm{~d}, \mathrm{~B}\end{array}$ & -44.57 & $\begin{array}{l}12.64 \pm \\
0.42^{c}, A\end{array}$ & -62.60 \\
\hline \multirow{5}{*}{$\begin{array}{c}\text { Salvia } \\
\text { officinalis }\end{array}$} & $\begin{array}{l}\text { freeze- } \\
\text { drying }\end{array}$ & $\begin{array}{l}57.98 \pm \\
1.27 \mathrm{k}, \mathrm{C}\end{array}$ & -8.66 & $\begin{array}{l}54.14 \pm \\
0.08^{\mathrm{h}, \mathrm{B}}\end{array}$ & -6.63 & $\begin{array}{l}41.82 \mathrm{t} \\
0.67 \mathrm{~h}, \mathrm{~A}\end{array}$ & -27.88 & $\begin{array}{l}41.21 \mathrm{t} \\
1.00^{\mathrm{j}, \mathrm{A}}\end{array}$ & -28.93 \\
\hline & $\begin{array}{l}\text { natural } \\
\text { drying }\end{array}$ & $\begin{array}{l}45.85 \pm \\
0.18 \mathrm{hi}, \mathrm{C}\end{array}$ & -27.77 & $\begin{array}{l}39.51 \pm \\
0.52 \mathrm{~g}, \mathrm{~B}\end{array}$ & -13.83 & $\begin{array}{l}39.06 \pm \\
0.93 \mathrm{~g}, \mathrm{~B}\end{array}$ & -14.80 & $\begin{array}{l}35.17 \pm \\
1.36^{\mathrm{i}, \mathrm{A}}\end{array}$ & -23.29 \\
\hline & $\begin{array}{l}\text { drying at } \\
30^{\circ} \mathrm{C}\end{array}$ & $\begin{array}{l}23.60 \pm \\
0.08^{\mathrm{a}, \mathrm{C}}\end{array}$ & -62.82 & $\begin{array}{l}19.80 \pm \\
0.32^{\mathrm{b}, \mathrm{B}}\end{array}$ & -16.10 & $\begin{array}{c}19.38 \pm \\
0.28^{\mathrm{d}}, \mathrm{AB}\end{array}$ & -17.91 & $\begin{array}{l}18.32 \pm \\
0.65^{\mathrm{f}, \mathrm{A}}\end{array}$ & -22.38 \\
\hline & $\begin{array}{c}\text { drying at } \\
40^{\circ} \mathrm{C}\end{array}$ & $\begin{array}{l}31.44 \pm \\
0.08 \mathrm{~d}, \mathrm{D}\end{array}$ & -50.47 & $\begin{array}{l}29.40 \pm \\
0.95^{\mathrm{f}, \mathrm{C}}\end{array}$ & -6.47 & $\begin{array}{l}24.82 \pm \\
0.09^{\mathrm{f}, \mathrm{B}}\end{array}$ & -21.04 & $\begin{array}{l}15.42 \pm \\
0.41 \mathrm{e}, \mathrm{A}\end{array}$ & -50.97 \\
\hline & $\begin{array}{c}\text { drying at } \\
50^{\circ} \mathrm{C}\end{array}$ & $\begin{array}{l}31.79 \pm \\
0.26^{\mathrm{d}}, \mathrm{D}\end{array}$ & -49.92 & $\begin{array}{l}23.56 \pm \\
0.17 \mathrm{~d}, \mathrm{C}\end{array}$ & -25.89 & $\begin{array}{l}14.60 \pm \\
0.18^{\mathrm{b}, \mathrm{B}}\end{array}$ & -54.08 & $\begin{array}{l}11.04 \pm \\
0.13^{\mathrm{b}, \mathrm{A}}\end{array}$ & -65.28 \\
\hline \multirow{5}{*}{$\begin{array}{l}\text { Salvia } \\
\text { sclarea }\end{array}$} & $\begin{array}{l}\text { freeze- } \\
\text { drying }\end{array}$ & $\begin{array}{l}71.04 \pm \\
0.23 \mathrm{~m}, \mathrm{D}\end{array}$ & -34.86 & $\begin{array}{l}54.63 \pm \\
1.11^{\mathrm{h}, \mathrm{C}}\end{array}$ & -23.10 & $\begin{array}{l}49.60 \pm \\
0.17^{\mathrm{i}, \mathrm{B}}\end{array}$ & -30.18 & $\begin{array}{l}28.85 \pm \\
0.19 \mathrm{~h}, \mathrm{~A}\end{array}$ & -59.38 \\
\hline & $\begin{array}{c}\text { natural } \\
\text { drying }\end{array}$ & $\begin{array}{l}46.68 \pm \\
0.55 \mathrm{ij}, \mathrm{D}\end{array}$ & -57.20 & $\begin{array}{l}23.62 \pm \\
0.07 \mathrm{~d}, \mathrm{C}\end{array}$ & -49.40 & $\begin{array}{l}19.56 \pm \\
0.09 \mathrm{~d}, \mathrm{~B}\end{array}$ & -58.10 & $\begin{array}{c}14.78 \pm \\
0.83 \mathrm{de}, \mathrm{A}\end{array}$ & -68.35 \\
\hline & $\begin{array}{c}\text { drying at } \\
30^{\circ} \mathrm{C}\end{array}$ & $\begin{array}{l}42.24 \pm \\
0.78 \mathrm{~g}, \mathrm{D}\end{array}$ & -61.27 & $\begin{array}{l}20.98 \pm \\
0.14^{c, c}\end{array}$ & -50.34 & $\begin{array}{l}19.71 \pm \\
0.18^{\mathrm{d}, \mathrm{B}}\end{array}$ & -53.35 & $\begin{array}{l}17.56 \pm \\
0.19^{\mathrm{f}, \mathrm{A}}\end{array}$ & -58.42 \\
\hline & $\begin{array}{c}\text { drying at } \\
40^{\circ} \mathrm{C}\end{array}$ & $\begin{array}{l}47.38 \pm \\
0.53 \mathrm{j}, \mathrm{C}\end{array}$ & -56.56 & $\begin{array}{l}22.41 \pm \\
0.26 \mathrm{~d}, \mathrm{~B}\end{array}$ & -52.71 & $\begin{array}{l}17.08 \pm \\
0.53^{c, A}\end{array}$ & -63.95 & $\begin{array}{l}16.91 \pm \\
0.53^{f, A}\end{array}$ & -64.31 \\
\hline & $\begin{array}{c}\text { drying at } \\
50^{\circ} \mathrm{C}\end{array}$ & $\begin{array}{l}45.16 \pm \\
1.42 \mathrm{~h}, \mathrm{D}\end{array}$ & -58.59 & $\begin{array}{l}23.39 \pm \\
0.07^{\mathrm{d}, \mathrm{C}}\end{array}$ & -48.20 & $\underset{\substack{9, \mathrm{~B} \\
\mathbf{a}}}{ \pm .14} 0.00$ & -79.77 & $\begin{array}{c}5.85 \pm 0.00 \\
\mathrm{a}, \mathrm{A}\end{array}$ & -87.05 \\
\hline
\end{tabular}

Results are expressed as mean $\pm \mathrm{SD}(n=3)$. DW—dry weight. Mean values with different letters $(\mathrm{a}-\mathrm{m})$ within the individual storage periods (columns) are statistically different $(p<0.05)$. Mean values with different letters (A-D) within the individual species of sage and drying methods (rows) are statistically different $(p<0.05) .{ }^{*}$ Changes in the content of total carotenoids with reference to fresh sage samples. ${ }^{* *}$ Changes in the content of total carotenoids with reference to sage samples directly after drying.

\subsection{The Content of Total Polyphenols}

In the fresh herbs, Salvia hispanica and Salvia officinalis were characterized by a significantly higher content of total polyphenols $\left(9.76 \mathrm{~g} \cdot 100 \mathrm{~g}^{-1} \mathrm{DW}\right.$ and $9.47 \mathrm{~g} \cdot 100 \mathrm{~g}^{-1} \mathrm{DW}$, respectively) in comparison to Salvia sclarea ( $\left.4.95 \mathrm{~g} \cdot 100 \mathrm{~g}^{-1} \mathrm{DW}\right)$ (Table 2). The drying method affected the level of these compounds significantly (Table 4). In the herbs Salvia hispanica and Salvia officinalis, an increase in the content of polyphenols was found in the samples after freeze-drying and natural drying. However, the highest content was observed after freeze-drying (12.89 g.100 g ${ }^{-1}$ DW in Salvia officinalis and $11.84 \mathrm{~g} \cdot 100 \mathrm{~g}^{-1} \mathrm{DW}$ in Salvia hispanica). In these herbs, a loss in polyphenolic compounds was found in samples after drying at $30^{\circ} \mathrm{C}, 40{ }^{\circ} \mathrm{C}$, and $50^{\circ} \mathrm{C}$. The lowest level of these compounds was determined in these herbs after drying at $50{ }^{\circ} \mathrm{C}\left(6.11 \mathrm{~g} \cdot 100 \mathrm{~g}^{-1} \mathrm{DW}\right.$ in Salvia officinalis and $5.49 \mathrm{~g} \cdot 100$ $\mathrm{g}^{-1} \mathrm{DW}$ in Salvia hispanica). However, for the Salvia sclarea samples, all drying methods caused an increase in the content of polyphenols. The greatest changes were found after natural drying $\left(8.52 \mathrm{~g} \cdot 100 \mathrm{~g}^{-1} \mathrm{DW}\right)$. Storage resulted in a decrease in levels of polyphenolic compounds in all the tested samples. The lowest levels of these compounds, caused by the highest loss, were found after 12 months of storage. 
Table 4. The content of total polyphenols in dried and stored sage of individual species.

\begin{tabular}{|c|c|c|c|c|c|c|c|c|c|}
\hline \multirow[b]{3}{*}{ Species } & \multirow[b]{3}{*}{$\begin{array}{l}\text { Drying } \\
\text { Method }\end{array}$} & \multicolumn{8}{|c|}{ Storage } \\
\hline & & \multicolumn{2}{|c|}{ Directly after Drying } & \multicolumn{2}{|c|}{ After 3 Months } & \multicolumn{2}{|c|}{ After 6 Months } & \multicolumn{2}{|c|}{ After 12 Months } \\
\hline & & $\begin{array}{c}\text { Total } \\
\text { Polyphenols } \\
{\left[\mathrm{g} \cdot 100 \mathrm{~g}^{-1}\right.} \\
\text { DW] }\end{array}$ & $\begin{array}{c}\text { Changes * } \\
{[\%]}\end{array}$ & $\begin{array}{c}\text { Total } \\
\text { Polyphenols } \\
{\left[\mathrm{g} \cdot 100 \mathrm{~g}^{-1}\right.} \\
\text { DW] }\end{array}$ & $\begin{array}{c}\text { Changes ** } \\
{[\%]}\end{array}$ & $\begin{array}{c}\text { Total } \\
\text { Polyphenols } \\
\text { [g.100 g } \\
\text { DW] }\end{array}$ & $\begin{array}{c}\text { Changes } * * \\
{[\%]}\end{array}$ & $\begin{array}{c}\text { Total } \\
\text { Polyphenols } \\
{\left[\mathrm{g} \cdot 100 \mathrm{~g}^{-1}\right.} \\
\text { DW] }\end{array}$ & $\underset{[\%]}{\text { Changes ** }}$ \\
\hline \multirow{5}{*}{$\begin{array}{c}\text { Salvia } \\
\text { hispanica }\end{array}$} & freeze-drying & $\underset{\text { hi, A }}{11.84 \pm 1.68}$ & 21.30 & $\begin{array}{c}10.83 \\
\mathrm{i}, \mathrm{A}\end{array}$ & -8.46 & $10.40 \pm \underset{\mathrm{i}, \mathrm{A}}{ \pm} 0.54$ & -12.14 & $10.71 \underset{\mathrm{f}, \mathrm{A}}{ \pm} 0.24$ & -9.49 \\
\hline & natural drying & $\begin{array}{c}10.79 \pm 0.07 \\
\text { gh, B }\end{array}$ & 10.59 & $10.71 \underset{\text { hi, B }}{ \pm} 0.24$ & -0.73 & $\underset{\mathrm{gh}, \mathrm{A}}{9.25 \pm 0.12}$ & -14.30 & $\begin{array}{c}9.23 \pm 0.47 \\
\mathrm{e}, \mathrm{A}\end{array}$ & -14.44 \\
\hline & drying at $30^{\circ} \mathrm{C}$ & $9.73 \underset{\mathrm{fg}, \mathrm{C}}{ \pm} 0.14$ & -0.28 & $\underset{\mathrm{g}, \mathrm{B}}{9.16 \pm 0.06}$ & -7.50 & $\underset{\mathrm{g}, \mathrm{A}}{8.82 \pm 0.10}$ & -10.99 & $8.57 \underset{d, A}{ \pm} 0.14$ & -13.51 \\
\hline & drying at $40^{\circ} \mathrm{C}$ & $9.66 \underset{f, D}{ \pm} 0.02$ & -0.97 & $\underset{\mathrm{g}, \mathrm{C}}{9.25 \pm 0.20}$ & -4.29 & $\underset{\mathrm{g}, \mathrm{B}}{8.73 \pm 0.05}$ & -9.68 & $\underset{d, A}{8.34 \pm 0.10}$ & -13.68 \\
\hline & drying at $50^{\circ} \mathrm{C}$ & $\begin{array}{c}5.49 \pm 0.15 \\
\mathrm{a}, \mathrm{C}\end{array}$ & -43.78 & $4.71 \underset{a, B}{ \pm 0} 0.05$ & -14.07 & $\underset{\mathrm{a}, \mathrm{B}}{4.58 \pm 0.01}$ & -16.55 & $\begin{array}{c}4.09 \pm 0.04 \\
\underset{a, A}{A}\end{array}$ & -25.42 \\
\hline \multirow{5}{*}{$\begin{array}{c}\text { Salvia } \\
\text { officinalis }\end{array}$} & freeze-drying & $12.89 \underset{\mathrm{i}, \mathrm{B}}{ \pm} 0.63$ & 36.11 & $12.09 \underset{\mathrm{j}, \mathrm{AB}}{ \pm} 0.25$ & -6.22 & $11.91 \underset{\mathrm{j}, \mathrm{AB}}{ \pm} 0.50$ & -7.60 & $\underset{\mathrm{f}, \mathrm{A}}{10.94 \pm 0.69}$ & -15.14 \\
\hline & natural drying & $12.03 \underset{i, C}{ \pm} 0.60$ & 27.02 & $\underset{\mathrm{h}, \mathrm{B}}{10.09 \pm 0.61}$ & -16.09 & $9.59 \underset{\mathrm{h}, \mathrm{B}}{ \pm} 0.38$ & -20.25 & $\underset{\mathrm{ds}, \mathrm{A}}{\mathbf{8}} \mathbf{0 . 3 3}$ & -32.11 \\
\hline & drying at $30^{\circ} \mathrm{C}$ & $\underset{\text { de, B }}{7.90 \pm 0.16}$ & -16.58 & $\begin{array}{c}7.32 \pm 0.54 \\
\mathrm{de}, \mathrm{AB}\end{array}$ & -7.35 & $6.81 \underset{\mathrm{f}, \mathrm{A}}{ \pm} 0.00$ & -13.74 & $6.64 \underset{c, A}{ \pm} 0.10$ & -16.01 \\
\hline & drying at $40^{\circ} \mathrm{C}$ & $\underset{\text { bcd, C }}{7.13 \pm 0.05}$ & -24.72 & $7.15 \underset{d, C}{ \pm} 0.01$ & 0.27 & $\begin{array}{c}6.19 \pm 0.16 \\
\text { cde, } B\end{array}$ & -13.23 & $\underset{b, A}{5.59} \underset{b}{ \pm} 0.19$ & -21.66 \\
\hline & drying at $50^{\circ} \mathrm{C}$ & $\begin{array}{c}6.11 \pm 0.07 \\
\mathrm{ab}, \mathrm{A}\end{array}$ & -35.49 & $6.11 \underset{\mathrm{bc}, \mathrm{A}}{ \pm} 0.47$ & 0.07 & $\begin{array}{c}5.95 \pm 0.04 \\
\mathrm{~cd}, \mathrm{~A}\end{array}$ & -2.66 & $\underset{\mathrm{b}, \mathrm{A}}{5.9 .13}$ & -2.80 \\
\hline \multirow{5}{*}{$\begin{array}{l}\text { Salvia } \\
\text { sclarea }\end{array}$} & freeze-drying & $\begin{array}{c}8.14 \pm 0.01 \\
\text { de, } C\end{array}$ & 64.50 & $7.89 \underset{\text { ef, },}{ \pm 0.15}$ & -3.05 & $\underset{\mathrm{cd}, \mathrm{B}}{6.12 \pm 0.21}$ & -24.79 & $\underset{\mathrm{b}, \mathrm{A}}{ \pm .89}$ & -27.63 \\
\hline & natural drying & $8.52 \underset{e, B}{ \pm} 0.01$ & 72.15 & $8.04 \underset{f, B}{ \pm} 0.16$ & -5.63 & $6.74 \underset{\text { ef, A }}{ \pm} 0.01$ & -20.86 & $\underset{\mathrm{b}, \mathrm{A}}{5.83 \pm 0.42}$ & -31.55 \\
\hline & drying at $30^{\circ} \mathrm{C}$ & $\begin{array}{c}7.69 \pm 0.13 \\
\text { cde, } B\end{array}$ & 55.51 & $\underset{\mathrm{cd}, \mathrm{A}}{6.70 \pm 0.16}$ & -12.89 & $6.51 \pm 0.50$ & -15.33 & $\underset{\mathrm{b}, \mathrm{A}}{5.92 \pm 0.34}$ & -23.04 \\
\hline & drying at $40^{\circ} \mathrm{C}$ & $\begin{array}{c}6.97 \pm 0.11 \\
\text { bcd, D }\end{array}$ & 40.90 & $5.66 \underset{b, C}{ \pm 0.04}$ & -18.83 & $\begin{array}{c}5.14 \underset{\mathrm{ab}, \mathrm{B}}{ \pm 0.04} \\
\end{array}$ & -26.27 & $\underset{\mathrm{a}, \mathrm{A}}{4.49 \pm 0.18}$ & -35.63 \\
\hline & drying at $50^{\circ} \mathrm{C}$ & $\begin{array}{c}6.70 \pm 0.27 \\
\mathrm{bc}, \mathrm{B}\end{array}$ & 35.45 & $6.33 \pm 0.05$ & -5.53 & $\begin{array}{c}5.70 \pm 0.10 \\
\mathrm{bc}, \mathrm{A}\end{array}$ & -14.99 & $\underset{\mathrm{b}, \mathrm{A}}{5.74 \pm 0.01}$ & -14.29 \\
\hline
\end{tabular}

Results are expressed as mean $\pm \mathrm{SD}(n=3)$. DW-dry weight. Mean values with different letters $(\mathrm{a}-\mathrm{j})$ within the individual storage periods (columns) are statistically different $(p<0.05)$. Mean values with different letters (A-D) within the individual species of sage and drying methods (rows) are statistically different $(p<0.05) .{ }^{*}$ Changes in the content of total polyphenols with reference to fresh sage samples. ${ }^{*}$ Changes in the content of total polyphenols with reference to sage samples directly after drying.

\subsection{Polyphenol Profile}

The following polyphenols were found: 4-hydroxybenzoic acid, caffeic acid, chlorogenic acid, ferulic acid, gallic acid, p-coumaric acid, rosmarinic acid, sinapinic acid, syringic acid, vanillic acid, acacetin, apigenin, catechin, epicatechin, hesperidin, hispidulin, isorhamnetin, kaempferol, luteolin, myricetin, naringin, quercetin, rutin, carnosic acid, and carnosol. The polyphenols detected in Salvia hispanica, Salvia officinalis, and Salvia sclarea, divided into phenolic acids, flavonoids, and phenolic diterpenes, are shown in Table 5, Table 6, and Table 7, respectively.

In the fresh herb of Salvia hispanica, 4-hydroxybenzoic acid, ferulic acid, syringic acid, acacetin, catechin, epicatechin, hispidulin, kaempferol, and quercetin were not identified. Rosmarinic acid (1358.80 mg.100 g $\left.{ }^{-1} \mathrm{DW}\right)$, sinapinic acid (196.71 mg.100 g $\left.{ }^{-1} \mathrm{DW}\right)$, naringin (199.07 mg.100 g $\left.{ }^{-1} \mathrm{DW}\right)$, and rutin $\left(197.59 \mathrm{mg} \cdot 100 \mathrm{~g}^{-1} \mathrm{DW}\right)$ were the dominant polyphenols in this species. In the fresh herb of Salvia officinalis, apigenin, catechin, and hispidulin were not detected. However, the highest contents of rosmarinic acid $\left(1488.28 \mathrm{mg} \cdot 100 \mathrm{~g}^{-1} \mathrm{DW}\right)$, hesperidin (346.43 mg.100 g $\left.{ }^{-1} \mathrm{DW}\right)$, naringin $\left(523.85 \mathrm{mg} \cdot 100 \mathrm{~g}^{-1} \mathrm{DW}\right)$, and rutin (317.77 $\left.\mathrm{mg} \cdot 100 \mathrm{~g}^{-1} \mathrm{DW}\right)$ were measured. In the fresh herb of Salvia sclarea, p-coumaric acid, acacetin, catechin, hispidulin, kaempferol, myricetin, and quercetin were not found. The following polyphenolic compounds were dominant in this species of sage: rosmarinic acid (346.32 mg.100 g $\left.{ }^{-1} \mathrm{DW}\right)$, rutin $\left(127.41 \mathrm{mg} \cdot 100 \mathrm{~g}^{-1} \mathrm{DW}\right)$, and carnosol (296.72 mg.100 $\left.\mathrm{g}^{-1} \mathrm{DW}\right)$. 
Table 5. Concentration of individual polyphenolic compounds in fresh, dried, and stored Salvia hispanica.

\begin{tabular}{|c|c|c|c|c|c|c|c|c|c|c|c|}
\hline \multirow{3}{*}{$\begin{array}{l}\text { Polyphenolic } \\
\text { Compounds }\end{array}$} & \multirow[b]{2}{*}{ Fresh } & \multicolumn{5}{|c|}{ Directly after Drying } & \multicolumn{5}{|c|}{ After 12 Months of Storage } \\
\hline & & $\begin{array}{l}\text { Freeze- } \\
\text { Drying }\end{array}$ & $\begin{array}{l}\text { Natural } \\
\text { Drying }\end{array}$ & $\begin{array}{l}\text { Drying } \\
\text { at } 30^{\circ} \mathrm{C}\end{array}$ & $\begin{array}{l}\text { Drying } \\
\text { at } 40^{\circ} \mathrm{C}\end{array}$ & $\begin{array}{l}\text { Drying } \\
\text { at } 50^{\circ} \mathrm{C}\end{array}$ & $\begin{array}{l}\text { Freeze- } \\
\text { Drying }\end{array}$ & $\begin{array}{l}\text { Natural } \\
\text { Drying }\end{array}$ & $\begin{array}{l}\text { Drying } \\
\text { at } 30^{\circ} \mathrm{C}\end{array}$ & $\begin{array}{l}\text { Drying } \\
\text { at } 40^{\circ} \mathrm{C}\end{array}$ & $\begin{array}{l}\text { Drying } \\
\text { at } 50^{\circ} \mathrm{C}\end{array}$ \\
\hline & \multicolumn{11}{|c|}{$\mathrm{mg} \cdot 100 \mathrm{~g}^{-1} \mathrm{DW}$} \\
\hline \multicolumn{12}{|l|}{ Phenolic } \\
\hline $\begin{array}{l}4- \\
\text { Hydroxybenzoic } \\
\text { acid }\end{array}$ & nd & $\begin{array}{c}22.74 \pm \\
0.22^{\mathrm{h}}\end{array}$ & $\begin{array}{l}7.44 \pm \\
0.08^{\mathrm{c}}\end{array}$ & $\begin{array}{r}16.19 \pm \\
0.02^{\mathrm{f}}\end{array}$ & $\begin{array}{c}12.61 \pm \\
0.00^{\mathrm{e}}\end{array}$ & $\begin{array}{l}5.40 \pm \\
0.00^{\mathrm{b}}\end{array}$ & $\begin{array}{c}17.90 \pm \\
0.11^{\mathrm{g}}\end{array}$ & $\begin{array}{l}4.89 \pm \\
0.10^{\mathrm{a}}\end{array}$ & $\begin{array}{r}11.32 \pm \\
0.21^{\mathrm{d}}\end{array}$ & $\begin{array}{c}12.63 \pm \\
0.19^{\mathrm{e}}\end{array}$ & $\begin{array}{l}4.62 \pm \\
0.04^{\mathrm{a}}\end{array}$ \\
\hline Caffeic acid & $\begin{array}{c}17.11 \pm \\
0.06^{\mathrm{h}}\end{array}$ & $\begin{array}{c}16.51 \pm \\
0.06^{\mathrm{g}}\end{array}$ & $\begin{array}{c}22.15 \pm \\
0.00^{\mathrm{i}}\end{array}$ & $\begin{array}{r}13.97 \pm \\
0.00^{\mathrm{f}}\end{array}$ & $\begin{array}{c}14.05 \pm \\
0.06^{\mathrm{f}}\end{array}$ & $\begin{array}{l}9.50 \pm \\
0.00^{c}\end{array}$ & $\begin{array}{c}12.01 \pm \\
0.23 \mathrm{e}^{\mathrm{e}}\end{array}$ & $\begin{array}{l}8.02 \pm \\
0.04^{b}\end{array}$ & $\begin{array}{r}11.51 \pm \\
0.06^{\mathrm{d}}\end{array}$ & $\begin{array}{r}11.56 \pm \\
0.00^{\mathrm{d}}\end{array}$ & $\begin{array}{l}6.85 \pm \\
0.00^{\mathrm{a}}\end{array}$ \\
\hline $\begin{array}{l}\text { Chlorogenic } \\
\text { acid }\end{array}$ & $\begin{array}{c}14.88 \pm \\
0.00 \mathrm{e}^{\mathrm{e}}\end{array}$ & $\begin{array}{r}23.74 \pm \\
0.16^{j}\end{array}$ & $\begin{array}{l}7.01 \pm \\
0.04^{\mathrm{c}}\end{array}$ & $\begin{array}{c}19.38 \pm \\
0.08 \mathrm{~g}\end{array}$ & $\begin{array}{c}15.67 \pm \\
0.02^{f}\end{array}$ & $\begin{array}{l}6.36 \pm \\
0.00^{a}\end{array}$ & $\begin{array}{r}22.31 \pm \\
0.32^{\mathrm{i}}\end{array}$ & $\begin{array}{l}6.85 \pm \\
0.50 \mathrm{bc}\end{array}$ & $\begin{array}{c}20.92 \pm \\
0.02^{\mathrm{h}}\end{array}$ & $\begin{array}{r}10.00 \pm \\
0.63^{\mathrm{d}}\end{array}$ & $\begin{array}{l}6.95 \pm \\
0.02{ }^{\mathrm{bc}}\end{array}$ \\
\hline Ferulic acid & nd & $\begin{array}{r}11.25 \pm \\
0.30^{\mathrm{f}}\end{array}$ & $\begin{array}{l}3.92 \pm \\
0.00^{\mathrm{b}}\end{array}$ & $\begin{array}{r}11.64 \underset{\mathrm{f}}{\mathrm{f}} \\
0.25^{\mathrm{f}}\end{array}$ & $\begin{array}{l}6.51 \pm \\
0.38^{c}\end{array}$ & $\begin{array}{l}2.39 \pm \\
0.02^{\mathrm{a}}\end{array}$ & $\begin{array}{l}7.28 \pm \\
0.21 \mathrm{~d}\end{array}$ & $\begin{array}{c}2.79 \pm \\
0.17^{\mathrm{a}}\end{array}$ & $\begin{array}{l}7.79 \pm \\
0.06^{\mathrm{e}}\end{array}$ & $\begin{array}{l}6.53 \pm \\
0.04^{\mathrm{c}}\end{array}$ & $\begin{array}{l}2.47 \pm \\
0.04^{\mathrm{a}}\end{array}$ \\
\hline Gallic acid & $\begin{array}{r}13.13^{ \pm} \\
0.00^{\mathrm{c}}\end{array}$ & $\begin{array}{l}4.84 \underset{\mathrm{t}}{0.04^{\mathrm{b}}} \\
\end{array}$ & $\begin{array}{r}17.19 \pm \\
0.16^{\mathrm{d}}\end{array}$ & nd & nd & nd & $\begin{array}{l}3.66 \pm \\
0.06^{\mathrm{a}}\end{array}$ & $\begin{array}{l}3.64 \pm \\
0.04^{\mathrm{a}}\end{array}$ & nd & nd & nd \\
\hline $\begin{array}{l}\text { p-Coumaric } \\
\text { acid }\end{array}$ & $\begin{array}{c}33.49^{ \pm} \\
0.06^{\mathrm{c}}\end{array}$ & $\begin{array}{c}48.97 \pm \\
0.04^{\mathrm{k}}\end{array}$ & $\begin{array}{c}43.62 \pm \\
0.00^{\mathrm{j}}\end{array}$ & $\begin{array}{r}41.44 \pm \\
0.044^{\mathrm{i}}\end{array}$ & $\begin{array}{c}40.63 \pm \\
0.06^{\mathrm{h}}\end{array}$ & $\begin{array}{c}34.03 \pm \\
0.02 \mathrm{e}^{\mathrm{e}}\end{array}$ & $\begin{array}{c}40.36 \pm \\
0.00^{g}\end{array}$ & $\begin{array}{c}22.73 \pm \\
0.03^{\mathrm{a}}\end{array}$ & $\begin{array}{c}33.74 \pm \\
0.04^{\mathrm{d}}\end{array}$ & $\begin{array}{c}39.15 \pm \\
0.06^{\mathrm{f}}\end{array}$ & $\begin{array}{c}30.48 \pm \\
0.02^{\mathrm{b}}\end{array}$ \\
\hline $\begin{array}{c}\text { Rosmarinic } \\
\text { acid }\end{array}$ & $\begin{array}{c}1358.80 \pm \\
1.18^{\mathrm{g}}\end{array}$ & $\begin{array}{c}1783.30 \pm \\
0.91^{\mathrm{k}}\end{array}$ & $\begin{array}{c}1769.02 \pm \\
1.88^{\mathrm{j}}\end{array}$ & $\begin{array}{c}1458.40 \pm \\
1.10^{\mathrm{h}}\end{array}$ & $\begin{array}{c}1315.27 \pm \\
1.68^{\mathrm{f}}\end{array}$ & $\begin{array}{c}200.31 \pm \\
0.21^{\mathrm{b}}\end{array}$ & $\begin{array}{c}1558.61^{\mathrm{i}} \pm \\
1.04^{\mathrm{i}}\end{array}$ & $\begin{array}{c}636.47 \pm \\
1.63^{c}\end{array}$ & $\begin{array}{c}1139.64 \pm \\
0.43^{\mathrm{e}}\end{array}$ & $\begin{array}{c}905.93 \pm \\
1.82^{\mathrm{d}}\end{array}$ & $\begin{array}{c}170.86 \pm \\
0.29^{\mathrm{a}}\end{array}$ \\
\hline Sinapinic acid & $\begin{array}{c}196.71 \pm \\
0.00^{\mathrm{h}}\end{array}$ & $\begin{array}{r}11.81 \underset{\mathrm{f}}{\mathrm{f}} \\
0.65^{\mathrm{f}}\end{array}$ & $\begin{array}{c}258.92 \pm \\
0.16^{\mathrm{i}}\end{array}$ & $\begin{array}{r}10.27 \pm \\
0.04 \mathrm{e}^{ \pm}\end{array}$ & $\begin{array}{l}6.56 \pm \\
0.00^{c}\end{array}$ & $\begin{array}{c}2.80 \pm \\
0.10^{a}\end{array}$ & $\begin{array}{l}9.53 \pm \\
0.02 \mathrm{~d}\end{array}$ & $\begin{array}{c}35.02 \mathrm{I} \\
0.03 \mathrm{~g}\end{array}$ & $\begin{array}{l}6.12 \pm \\
0.19 \mathrm{bc}\end{array}$ & $\begin{array}{l}5.77 \stackrel{ \pm}{\mathrm{b}} \\
0.25^{\mathrm{b}}\end{array}$ & $\begin{array}{l}2.80 \pm \\
0.00^{\mathrm{a}}\end{array}$ \\
\hline Syringic acid & nd & $\begin{array}{c}11.48 \pm \\
0.06^{\mathrm{h}}\end{array}$ & $\begin{array}{c}5.71 \pm \\
0.08^{\mathrm{e}}\end{array}$ & $\begin{array}{l}8.79 \pm \\
0.12 \mathrm{~g}\end{array}$ & $\begin{array}{l}4.60 \pm \\
0.02^{\mathrm{b}}\end{array}$ & $\begin{array}{l}4.95 \pm \\
0.02^{\mathrm{c}}\end{array}$ & $\begin{array}{l}5.51 \mathrm{t} \\
0.11^{\mathrm{d}}\end{array}$ & $\begin{array}{l}3.10 \pm \\
0.11^{\mathrm{a}}\end{array}$ & $\begin{array}{l}6.68 \pm \\
0.02^{\mathrm{f}}\end{array}$ & $\begin{array}{l}4.74 \pm \\
0.04^{\mathrm{b}}\end{array}$ & $\begin{array}{l}4.60 \pm \\
0.02^{\mathrm{b}}\end{array}$ \\
\hline Vanillic acid & $\begin{array}{l}5.78 \pm \\
0.00^{f}\end{array}$ & $\begin{array}{l}5.30 \pm \\
0.02^{\mathrm{d}}\end{array}$ & $\begin{array}{r}7.50 \pm \\
0.00^{\mathrm{i}}\end{array}$ & $\begin{array}{l}6.14 \pm \\
0.04 \mathrm{~g}\end{array}$ & $\begin{array}{l}6.53 \pm \\
0.02^{h}\end{array}$ & $\begin{array}{l}5.42 \pm \\
0.00^{\mathrm{e}}\end{array}$ & $\begin{array}{l}4.33{ }^{ \pm} \\
0.04^{c}\end{array}$ & $\begin{array}{l}3.88 \pm \\
0.02^{\mathrm{b}}\end{array}$ & $\begin{array}{l}6.18 \pm \\
0.02 \mathrm{~g}\end{array}$ & $\begin{array}{l}6.44 \pm \\
0.12^{\mathrm{h}}\end{array}$ & $\begin{array}{l}3.45 \pm \\
0.00^{\mathrm{a}}\end{array}$ \\
\hline \multicolumn{12}{|l|}{ Flavonoids: } \\
\hline Apigenin & $\begin{array}{l}2.93 \pm \\
0.06^{\mathrm{h}}\end{array}$ & $\begin{array}{l}1.38 \pm \\
0.02 \mathrm{~g}\end{array}$ & $\begin{array}{l}1.04 \pm \\
0.00^{\mathrm{e}}\end{array}$ & $\begin{array}{l}0.57 \pm \\
0.02^{d}\end{array}$ & $\begin{array}{l}0.48 \pm \\
0.04^{\mathrm{c}}\end{array}$ & $\begin{array}{l}0.28 \pm \\
0.02^{\mathrm{b}}\end{array}$ & $\begin{array}{l}1.22 \pm \\
0.04^{\mathrm{f}}\end{array}$ & $\begin{array}{l}0.62 \pm \\
0.04^{\mathrm{d}}\end{array}$ & $\begin{array}{l}0.33 \pm \\
0.02^{\mathrm{b}}\end{array}$ & $\begin{array}{l}0.12 \pm \\
0.00^{\mathrm{a}}\end{array}$ & $\begin{array}{l}0.15 \pm \\
0.00^{\mathrm{a}}\end{array}$ \\
\hline Epicatechin & nd & $\begin{array}{c}15.26 \pm \\
1.30 \mathrm{~d}\end{array}$ & $\begin{array}{c}26.19 \pm \\
0.82 \mathrm{~g}\end{array}$ & $\begin{array}{c}50.90 \pm \\
0.70^{j}\end{array}$ & $\begin{array}{c}34.18 \pm \\
1.31^{\mathrm{i}}\end{array}$ & $\begin{array}{c}28.62 \pm \\
0.29^{\mathrm{h}}\end{array}$ & $\begin{array}{c}10.34 \pm \\
0.32^{\mathrm{a}}\end{array}$ & $\begin{array}{c}17.47 \pm \\
0.90^{\mathrm{e}}\end{array}$ & $\begin{array}{c}21.90 \pm \\
0.06^{\mathrm{f}}\end{array}$ & $\begin{array}{l}12.02 \pm \\
0.50^{\mathrm{ab}}\end{array}$ & $\begin{array}{r}12.89 \pm \\
0.13^{\mathrm{c}}\end{array}$ \\
\hline Hesperidin & $\begin{array}{c}70.21 \pm \\
0.87^{\mathrm{a}}\end{array}$ & $\begin{array}{c}288.22 \pm \\
0.40^{\mathrm{h}}\end{array}$ & $\begin{array}{c}231.92 \pm \\
7.51 \mathrm{~g}\end{array}$ & $\begin{array}{l}96.31 \pm \\
2.30 \mathrm{~d}\end{array}$ & $\begin{array}{c}96.24 \pm \\
1.58^{\mathrm{d}}\end{array}$ & $\begin{array}{c}70.98 \pm \\
1.17^{\mathrm{a}}\end{array}$ & $\begin{array}{c}165.28 \pm \\
10.76^{\mathrm{f}}\end{array}$ & $\begin{array}{c}110.83 \pm \\
8.20^{\mathrm{e}}\end{array}$ & $\begin{array}{l}93.85 \pm \\
2.87 \mathrm{~cd}\end{array}$ & $\begin{array}{c}84.09 \pm \\
4.38 \mathrm{bc}\end{array}$ & $\begin{array}{c}79.87 \pm \\
0.87 \mathrm{ab}\end{array}$ \\
\hline Hispidulin & nd & $\begin{array}{c}14.24 \pm \\
0.13^{\mathrm{f}}\end{array}$ & nd & $\begin{array}{l}6.422^{ \pm} \\
0.02^{c}\end{array}$ & $\begin{array}{l}5.79 \pm \\
0.06^{\mathrm{a}}\end{array}$ & $\begin{array}{l}9.61 \pm \\
0.04^{d}\end{array}$ & $\begin{array}{c}13.81 \pm \\
0.02 \mathrm{e}^{\mathrm{e}}\end{array}$ & nd & $\begin{array}{c}6.06 \pm \\
0.23^{\mathrm{b}}\end{array}$ & $\begin{array}{l}5.99 \pm \\
0.02^{\mathrm{ab}}\end{array}$ & $\begin{array}{l}9.74 \pm \\
0.04^{\mathrm{d}}\end{array}$ \\
\hline Isorhamnetin & $\begin{array}{c}15.80^{ \pm} \\
0.06^{\mathrm{c}}\end{array}$ & $\begin{array}{l}0.99 \pm \\
0.06 \mathrm{ab}\end{array}$ & $\begin{array}{c}17.02 \pm \\
1.39^{\mathrm{d}}\end{array}$ & $\begin{array}{c}0.51 \pm \\
0.04^{\mathrm{a}}\end{array}$ & $\begin{array}{c}0.44 \pm \\
0.00^{\mathrm{a}}\end{array}$ & $\begin{array}{c}0.71 \pm \\
0.04^{\mathrm{a}}\end{array}$ & $\begin{array}{c}0.67 \pm \\
0.02^{\mathrm{a}}\end{array}$ & $\begin{array}{l}1.89 \pm \\
0.22^{\mathrm{b}}\end{array}$ & $\begin{array}{c}0.16 \pm \\
0.02^{\mathrm{a}}\end{array}$ & $\begin{array}{c}0.38 \pm \\
0.04^{\mathrm{a}}\end{array}$ & $\begin{array}{c}0.09 \pm \\
0.00^{\mathrm{a}}\end{array}$ \\
\hline Kaempferol & nd & $\begin{array}{l}5.26 \pm \\
0.00^{\mathrm{h}}\end{array}$ & $\begin{array}{l}2.37 \pm \\
0.24^{\mathrm{b}}\end{array}$ & $\begin{array}{l}3.70 \pm \\
0.00^{\mathrm{e}}\end{array}$ & $\begin{array}{l}3.73 \pm \\
0.04 \mathrm{e}\end{array}$ & $\begin{array}{l}4.99 \pm \\
0.04 \mathrm{~g}\end{array}$ & $\begin{array}{l}4.97 \pm \\
0.02 \mathrm{~g}\end{array}$ & $\begin{array}{l}1.89 \pm \\
0.01^{\mathrm{a}}\end{array}$ & $\begin{array}{l}3.41 \pm \\
0.06^{\mathrm{d}}\end{array}$ & $\begin{array}{l}3.15 \pm \\
0.02^{c}\end{array}$ & $\begin{array}{l}4.53 \pm \\
0.00^{\mathrm{f}}\end{array}$ \\
\hline Luteolin & $\begin{array}{l}9.28 \pm \\
0.37^{\mathrm{e}}\end{array}$ & $\begin{array}{c}19.79 \\
0.13^{\mathrm{j}}\end{array}$ & $\begin{array}{c}12.40 \pm \\
0.41^{\mathrm{f}}\end{array}$ & $\begin{array}{c}13.19 \pm \\
0.08^{\mathrm{g}}\end{array}$ & $\begin{array}{c}14.14 \pm \\
0.04^{\mathrm{h}}\end{array}$ & $\begin{array}{c}14.98 \pm \\
0.08^{\mathrm{i}}\end{array}$ & $\begin{array}{l}4.40 \pm \\
0.08^{\mathrm{c}}\end{array}$ & $\begin{array}{l}8.73 \pm \\
0.01 \mathrm{~d}\end{array}$ & $\begin{array}{l}2.82 \pm \\
0.16^{\mathrm{a}}\end{array}$ & $\begin{array}{l}3.54 \pm \\
0.10^{\mathrm{b}}\end{array}$ & $\begin{array}{l}3.92 \pm \\
0.08^{b}\end{array}$ \\
\hline Myricetin & $\begin{array}{c}25.91 \pm \\
0.00^{\mathrm{b}}\end{array}$ & $\begin{array}{c}73.43 \pm \\
0.14^{\mathrm{h}}\end{array}$ & $\begin{array}{c}34.44^{ \pm} \\
0.08^{\mathrm{c}}\end{array}$ & $\begin{array}{r}36.29 \pm \\
0.00 \mathrm{~d}\end{array}$ & $\begin{array}{c}34.84 \pm \\
1.13^{\mathrm{c}}\end{array}$ & $\begin{array}{r}41.41 \pm \\
0.10^{\mathrm{f}}\end{array}$ & $\begin{array}{c}67.58 \pm \\
0.04 \mathrm{~g}\end{array}$ & $\begin{array}{c}23.47 \pm \\
0.05^{\mathrm{a}}\end{array}$ & $\begin{array}{c}34.24^{ \pm} \\
0.21^{\mathrm{c}}\end{array}$ & $\begin{array}{c}34.84 \pm \\
0.02^{\mathrm{c}}\end{array}$ & $\begin{array}{c}39.29 \pm \\
0.11^{\mathrm{e}}\end{array}$ \\
\hline Naringin & $\begin{array}{c}199.07 \pm \\
0.87^{\mathrm{d}}\end{array}$ & $\begin{array}{c}309.70 \pm \\
0.26^{\mathrm{h}}\end{array}$ & $\begin{array}{c}294.69 \pm \\
10.28 \mathrm{~g}\end{array}$ & $\begin{array}{c}223.92 \pm \\
5.62^{\mathrm{f}}\end{array}$ & $\begin{array}{c}225.10 \pm \\
0.08^{\mathrm{f}}\end{array}$ & $\begin{array}{c}212.95 \pm \\
0.91 \mathrm{e}^{ \pm}\end{array}$ & $\begin{array}{c}291.40 \pm \\
0.34 \mathrm{~g}\end{array}$ & $\begin{array}{c}143.23 \pm \\
3.08^{\mathrm{a}}\end{array}$ & $\begin{array}{c}173.93 \pm \\
7.07^{\mathrm{b}}\end{array}$ & $\begin{array}{c}175.57 \pm \\
0.23 \mathrm{bc}\end{array}$ & $\begin{array}{c}184.91 \pm \\
0.04^{\mathrm{c}}\end{array}$ \\
\hline Quercetin & nd & $\begin{array}{c}4.10 \pm \\
0.27^{\mathrm{c}}\end{array}$ & $\begin{array}{c}8.83 \pm \\
0.73^{\mathrm{e}}\end{array}$ & $\begin{array}{l}6.43 \pm \\
0.04^{\mathrm{d}}\end{array}$ & $\begin{array}{l}9.82 \pm \\
0.06^{\mathrm{f}}\end{array}$ & $\begin{array}{l}4.79 \pm \\
0.42^{\mathrm{c}}\end{array}$ & $\begin{array}{c}2.18 \pm \\
0.12^{a}\end{array}$ & $\begin{array}{l}4.17^{ \pm} \\
0.05^{\mathrm{c}}\end{array}$ & $\begin{array}{c}3.32 \pm \\
0.04^{\mathrm{b}}\end{array}$ & $\begin{array}{l}5.98 \pm \\
0.21 \mathrm{~d}\end{array}$ & $\begin{array}{c}4.31 \pm \\
0.10^{c}\end{array}$ \\
\hline Rutin & $\begin{array}{c}197.59 \pm \\
0.74^{\mathrm{c}}\end{array}$ & $\begin{array}{c}314.09 \pm \\
1.73 \mathrm{~g}\end{array}$ & $\begin{array}{c}317.42 \pm \\
3.59 \mathrm{~g}\end{array}$ & $\begin{array}{c}272.39 \pm \\
4.98 \mathrm{e}^{ \pm}\end{array}$ & $\begin{array}{c}266.05 \pm \\
1.66^{\mathrm{e}}\end{array}$ & $\begin{array}{c}175.21 \pm \\
1.93^{\mathrm{b}}\end{array}$ & $\begin{array}{c}291.36 \pm \\
4.33^{\mathrm{f}}\end{array}$ & $\begin{array}{c}143.86 \pm \\
4.57^{\mathrm{a}}\end{array}$ & $\begin{array}{c}262.91 \pm \\
6.59 \mathrm{e}\end{array}$ & $\begin{array}{c}231.90 \pm \\
10.99 \mathrm{~d}\end{array}$ & $\begin{array}{c}168.51 \pm \\
0.42^{\mathrm{b}}\end{array}$ \\
\hline \multicolumn{12}{|l|}{$\begin{array}{c}\text { Phenolic } \\
\text { diterpenes: }\end{array}$} \\
\hline Carnosic acid & $\begin{array}{r}17.90 \pm \\
0.19^{\mathrm{c}}\end{array}$ & $\begin{array}{c}34.29 \pm \\
0.23 \mathrm{~g}\end{array}$ & $\begin{array}{c}53.42 \pm \\
2.77^{\mathrm{h}}\end{array}$ & $\begin{array}{c}20.92 \pm \\
1.10^{\mathrm{d}}\end{array}$ & $\begin{array}{c}29.26 \pm \\
0.23^{\mathrm{f}}\end{array}$ & $\begin{array}{r}16.37^{ \pm} \\
0.37^{\mathrm{c}}\end{array}$ & $\begin{array}{c}24.63 \pm \\
0.93^{\mathrm{e}}\end{array}$ & $\begin{array}{c}10.03 \pm \\
1.55^{\mathrm{b}}\end{array}$ & $\begin{array}{l}7.92 \pm \\
0.17 \mathrm{ab}\end{array}$ & $\begin{array}{l}18.61 \pm \\
1.01 \mathrm{~cd}\end{array}$ & $\begin{array}{c}5.69 \pm \\
0.31^{\mathrm{a}}\end{array}$ \\
\hline Carnosol & $\begin{array}{c}128.60 \pm \\
0.12^{\mathrm{h}}\end{array}$ & $\begin{array}{c}47.82 \pm \\
0.65^{\mathrm{g}}\end{array}$ & $\begin{array}{l}41.31 \pm \\
0.16^{\text {de }}\end{array}$ & $\begin{array}{c}44.30 \pm \\
0.27^{\mathrm{f}}\end{array}$ & $\begin{array}{c}42.11 \pm \\
0.43^{\mathrm{e}}\end{array}$ & $\begin{array}{l}40.12 \mathrm{t} \\
0.91 \mathrm{~cd}\end{array}$ & $\begin{array}{c}42.61 \pm \\
0.49^{\mathrm{e}}\end{array}$ & $\begin{array}{c}33.04 \pm \\
1.11^{\mathrm{b}}\end{array}$ & $\begin{array}{c}41.46 \pm \\
0.65^{\mathrm{e}}\end{array}$ & $\begin{array}{r}39.89 \pm \\
0.12^{\mathrm{c}}\end{array}$ & $\begin{array}{c}22.67 \pm \\
0.04^{\mathrm{a}}\end{array}$ \\
\hline
\end{tabular}

Results are expressed as mean \pm SD $(n=3)$. DW-dry weight; nd-not determined. Mean values with different letters $(\mathrm{a}-\mathrm{k})$ within the individual rows are statistically different $(p<0.05)$.

The results indicated that the method of drying significantly affected the level of individual polyphenolic compounds. Furthermore, differences in the polyphenol profiles before and after drying were found. In the herb of Salvia hispanica after drying, 4-hydroxybenzoic acid, ferulic acid, syringic acid, epicatechin, hispidulin, kaempferol, and quercetin were additionally determined. On the other hand, gallic acid was detected only in the fresh sample, after freeze-drying, and after natural drying. In samples of Salvia officinalis after drying, apigenin (with the exception of the sample after natural drying), catechin, and hispidulin were additionally identified. However, in the dried herb of Salvia sclarea, pcoumaric acid, acacetin, kaempferol, myricetin, and quercetin were detected (the exceptions were p-coumaric acid, kaempferol, and quercetin in the sample after natural drying). 
Table 6. Concentration of individual polyphenolic compounds in fresh, dried, and stored Salvia officinalis.

\begin{tabular}{|c|c|c|c|c|c|c|c|c|c|c|c|}
\hline \multirow{3}{*}{$\begin{array}{l}\text { Polyphenolic } \\
\text { Compounds }\end{array}$} & \multirow[b]{2}{*}{ Fresh } & \multicolumn{5}{|c|}{ Directly after Drying } & \multicolumn{5}{|c|}{ After 12 Months of Storage } \\
\hline & & $\begin{array}{l}\text { Freeze- } \\
\text { Drying }\end{array}$ & $\begin{array}{l}\text { Natural } \\
\text { Drying }\end{array}$ & $\begin{array}{l}\text { Drying } \\
\text { at } 30^{\circ} \mathrm{C}\end{array}$ & $\begin{array}{l}\text { Drying } \\
\text { at } 40^{\circ} \mathrm{C}\end{array}$ & $\begin{array}{l}\text { Drying } \\
\text { at } 50^{\circ} \mathrm{C}\end{array}$ & $\begin{array}{l}\text { Freeze- } \\
\text { Drying }\end{array}$ & $\begin{array}{l}\text { Natural } \\
\text { Drying }\end{array}$ & $\begin{array}{l}\text { Drying } \\
\text { at } 30^{\circ} \mathrm{C}\end{array}$ & $\begin{array}{l}\text { Drying } \\
\text { at } 40^{\circ} \mathrm{C}\end{array}$ & $\begin{array}{l}\text { Drying } \\
\text { at } 50^{\circ} \mathrm{C}\end{array}$ \\
\hline & \multicolumn{11}{|c|}{$\mathrm{mg} \cdot 100 \mathrm{~g}^{-1} \mathrm{DW}$} \\
\hline \\
\hline $\begin{array}{c}\text { Acids: } \\
4- \\
\text { Hydroxybenzoic } \\
\text { acid }\end{array}$ & $\begin{array}{l}5.86 \pm \\
0.12^{\mathrm{ef}}\end{array}$ & $\begin{array}{c}31.01 \pm \\
1.26^{\mathrm{h}}\end{array}$ & $\begin{array}{c}30.09 \pm \\
0.83^{\mathrm{h}}\end{array}$ & $\begin{array}{l}6.28 \pm \\
0.08^{\mathrm{f}}\end{array}$ & $\begin{array}{l}4.02 \pm \\
0.02 \mathrm{bcd}\end{array}$ & $\begin{array}{l}4.98 \pm \\
0.02 \mathrm{de}\end{array}$ & $\begin{array}{c}10.22 \pm \\
0.08^{\mathrm{g}}\end{array}$ & $\begin{array}{l}4.71 \pm \\
0.29 \mathrm{~cd}\end{array}$ & $\begin{array}{l}2.09 \pm \\
0.02^{\mathrm{a}}\end{array}$ & $\begin{array}{l}3.36 \pm \\
0.00^{\mathrm{b}}\end{array}$ & $\begin{array}{l}3.68 \pm \\
0.04^{\mathrm{bc}}\end{array}$ \\
\hline Caffeic acid & $\begin{array}{r}33.97 \pm \\
0.24^{\mathrm{f}}\end{array}$ & $\begin{array}{c}80.07 \pm \\
2.25^{\mathrm{i}}\end{array}$ & $\begin{array}{c}45.83 \pm \\
0.58^{\mathrm{h}}\end{array}$ & $\begin{array}{c}36.79 \pm \\
0.11^{\mathrm{g}}\end{array}$ & $\begin{array}{r}24.53 \pm \\
0.04^{\mathrm{d}}\end{array}$ & $\begin{array}{l}23.72 \pm \\
0.06^{\mathrm{cd}}\end{array}$ & $\begin{array}{c}36.52 \pm \\
0.19 \mathrm{~g}\end{array}$ & $\begin{array}{c}18.16 \pm \\
0.02^{\mathrm{a}}\end{array}$ & $\begin{array}{c}29.80 \pm \\
0.02 \mathrm{e}^{\mathrm{e}}\end{array}$ & $\begin{array}{l}22.55 \pm \\
0.04{ }^{\mathrm{bc}}\end{array}$ & $\begin{array}{l}21.5 \pm \\
0.52^{\mathrm{b}}\end{array}$ \\
\hline $\begin{array}{l}\text { Chlorogenic } \\
\text { acid }\end{array}$ & $\begin{array}{c}44.23 \pm \\
0.12 \mathrm{e}^{\mathrm{e}}\end{array}$ & $\begin{array}{c}62.72 \pm \\
3.35 \mathrm{~g}\end{array}$ & $\begin{array}{l}56.84 \pm \\
2.57^{\mathrm{f}}\end{array}$ & $\begin{array}{r}15.04 \pm \\
0.06^{\mathrm{c}}\end{array}$ & $\begin{array}{c}11.68 \pm \\
0.02^{\mathrm{b}}\end{array}$ & $\begin{array}{l}12.55 \pm \\
0.76^{\mathrm{bc}}\end{array}$ & $\begin{array}{r}26.99 \pm \\
0.04^{\mathrm{d}}\end{array}$ & $\begin{array}{c}13.60 \pm \\
0.49 \mathrm{bc}\end{array}$ & $\begin{array}{l}12.08 \pm \\
0.19^{\mathrm{bc}}\end{array}$ & $\begin{array}{c}11.42 \pm \\
0.04^{\mathrm{b}}\end{array}$ & $\begin{array}{l}5.54 \pm \\
0.06^{\mathrm{a}}\end{array}$ \\
\hline Ferulic acid & $\begin{array}{c}12.24 \pm \\
0.00^{\mathrm{a}}\end{array}$ & $\begin{array}{c}58.59 \pm \\
0.15^{\mathrm{h}}\end{array}$ & $\begin{array}{r}23.77 \pm \\
0.33^{\mathrm{d}}\end{array}$ & $\begin{array}{c}48.33 \pm \\
0.04 \mathrm{~g}\end{array}$ & $\begin{array}{c}28.35 \pm \\
0.10^{\mathrm{e}}\end{array}$ & $\begin{array}{r}24.31 \pm \\
0.16^{\mathrm{d}}\end{array}$ & $\begin{array}{c}25.09 \pm \\
1.28^{\mathrm{d}}\end{array}$ & $\begin{array}{c}14.91 \pm \\
1.00^{\mathrm{b}}\end{array}$ & $\begin{array}{c}33.33 \pm \\
0.14^{\mathrm{f}}\end{array}$ & $\begin{array}{c}19.57 \pm \\
0.00^{c}\end{array}$ & $\begin{array}{r}19.04 \pm \\
1.04^{\mathrm{c}}\end{array}$ \\
\hline Gallic acid & $\begin{array}{c}11.47 \pm \\
0.24^{\mathrm{a}}\end{array}$ & $\begin{array}{c}63.88 \pm \\
0.75 \mathrm{~g}\end{array}$ & $\begin{array}{c}16.74 \pm \\
0.00^{\mathrm{b}}\end{array}$ & $\begin{array}{l}30.06 \pm \\
0.02^{\mathrm{cd}}\end{array}$ & $\begin{array}{c}31.70 \pm \\
0.06^{\mathrm{d}}\end{array}$ & $\begin{array}{l}45.70 \pm \\
2.69^{\mathrm{e}}\end{array}$ & $\begin{array}{l}59.62 \pm \\
0.04 \mathrm{f}\end{array}$ & $\begin{array}{r}15.91 \pm \\
0.14^{\mathrm{b}}\end{array}$ & $\begin{array}{l}30.16 \pm \\
0.44^{\mathrm{cd}}\end{array}$ & $\begin{array}{r}29.25 \pm \\
0.10^{c}\end{array}$ & $\begin{array}{c}44.64 \pm \\
0.02 \mathrm{e}^{\mathrm{e}}\end{array}$ \\
\hline $\begin{array}{l}\text { p-Coumaric } \\
\text { acid }\end{array}$ & $\begin{array}{l}4.83 \pm \\
0.00 \mathrm{de}\end{array}$ & $\begin{array}{l}5.22 \pm \\
0.39 \text { ef }\end{array}$ & $\begin{array}{l}5.62 \pm \\
0.50^{\mathrm{f}}\end{array}$ & $\begin{array}{l}5.03 \pm \\
0.02 \mathrm{de}\end{array}$ & $\begin{array}{l}3.54 \pm \\
0.08^{c}\end{array}$ & $\begin{array}{l}2.15 \pm \\
0.02^{a}\end{array}$ & $\begin{array}{l}3.35 \pm \\
0.21 \mathrm{bc}\end{array}$ & $\begin{array}{l}2.05 \pm \\
0.07^{\mathrm{a}}\end{array}$ & $\begin{array}{l}4.69 \pm \\
0.00^{\mathrm{d}}\end{array}$ & $\begin{array}{l}2.94 \pm \\
0.00^{\mathrm{b}}\end{array}$ & $\begin{array}{l}2.13 \pm \\
0.02^{a}\end{array}$ \\
\hline $\begin{array}{l}\text { Rosmarinic } \\
\text { acid }\end{array}$ & $\begin{array}{c}1488.28 \pm \\
17.50^{\mathrm{f}}\end{array}$ & $\begin{array}{c}3479.39 \pm \\
1.66^{\mathrm{i}}\end{array}$ & $\begin{array}{c}2016.88 \pm \\
28.89^{\mathrm{h}}\end{array}$ & $\begin{array}{c}1341.99 \pm \\
1.46^{\mathrm{e}}\end{array}$ & $\begin{array}{c}1231.21 \pm \\
0.78^{\mathrm{d}}\end{array}$ & $\begin{array}{c}1167.06 \pm \\
0.04^{\mathrm{c}}\end{array}$ & $\begin{array}{c}1832.81 \pm \\
77.86^{\mathrm{g}}\end{array}$ & $\begin{array}{c}913.8 \pm \\
0.55^{\mathrm{a}}\end{array}$ & $\begin{array}{c}1177.83 \pm \\
0.65^{\mathrm{cd}}\end{array}$ & $\begin{array}{c}1078.66 \pm \\
0.34^{\mathrm{b}}\end{array}$ & $\begin{array}{c}1123.51 \pm \\
15.71 \mathrm{bc}\end{array}$ \\
\hline Sinapinic acid & $\begin{array}{c}10.09 \pm \\
0.00 \mathrm{~d}\end{array}$ & $\begin{array}{c}52.80 \pm \\
0.11^{\mathrm{h}}\end{array}$ & $\begin{array}{c}17.21 \pm \\
1.16^{\mathrm{f}}\end{array}$ & $\begin{array}{c}19.46 \pm \\
0.10 \mathrm{~g}\end{array}$ & $\begin{array}{c}11.63 \pm \\
0.10^{\mathrm{e}}\end{array}$ & $\begin{array}{l}9.17 \pm \\
0.10^{\mathrm{d}}\end{array}$ & $\begin{array}{l}9.49 \pm \\
0.56^{\mathrm{d}}\end{array}$ & $\begin{array}{l}9.27 \pm \\
0.31 \mathrm{~d}\end{array}$ & $\begin{array}{l}7.21 \pm \\
0.02^{\mathrm{c}}\end{array}$ & $\begin{array}{l}4.68 \pm \\
0.12 \mathrm{~b}\end{array}$ & $\begin{array}{l}3.72 \pm \\
0.02^{\mathrm{a}}\end{array}$ \\
\hline Syringic acid & $\begin{array}{c}19.66 \pm \\
0.24^{\mathrm{b}}\end{array}$ & $\begin{array}{c}49.23 \pm \\
0.06^{\mathrm{i}}\end{array}$ & $\begin{array}{r}49.40 \pm \\
2.81^{\mathrm{i}}\end{array}$ & $\begin{array}{c}30.83 \pm \\
0.04 \mathrm{~g}\end{array}$ & $\begin{array}{c}25.82 \mathrm{I} \\
0.43^{\mathrm{ef}}\end{array}$ & $\begin{array}{r}27.55 \pm \\
0.65^{\mathrm{f}}\end{array}$ & $\begin{array}{c}44.69 \pm \\
1.34^{\mathrm{h}}\end{array}$ & $\begin{array}{c}13.13 \pm \\
0.09^{\mathrm{a}}\end{array}$ & $\begin{array}{l}23.82 \pm \\
0.04 \mathrm{de}\end{array}$ & $\begin{array}{l}21.11 \pm \\
0.02^{\mathrm{bc}}\end{array}$ & $\begin{array}{l}23.10 \pm \\
0.00^{\mathrm{cd}}\end{array}$ \\
\hline Vanillic acid & $\begin{array}{r}11.98 \pm \\
0.00^{\mathrm{d}}\end{array}$ & $\begin{array}{c}31.08 \pm \\
0.17^{\mathrm{h}}\end{array}$ & $\begin{array}{c}15.80 \pm \\
0.66^{\mathrm{e}}\end{array}$ & $\begin{array}{r}16.56 \pm \\
0.15^{\mathrm{f}}\end{array}$ & $\begin{array}{r}11.04 \pm \\
0.06^{\mathrm{c}}\end{array}$ & $\begin{array}{r}11.43{ }^{ \pm} \\
0.04^{\mathrm{c}}\end{array}$ & $\begin{array}{c}17.90 \pm \\
0.08^{\mathrm{g}}\end{array}$ & $\begin{array}{c}7.78 \pm \\
0.05^{\mathrm{a}}\end{array}$ & $\begin{array}{r}11.10 \pm \\
0.06^{\mathrm{c}}\end{array}$ & $\begin{array}{l}9.80 \pm \\
0.02^{\mathrm{b}}\end{array}$ & $\begin{array}{c}10.11 \pm \\
0.23^{\mathrm{b}}\end{array}$ \\
\hline \multicolumn{12}{|l|}{ Flavonoids: } \\
\hline Acacetin & $\begin{array}{c}26.98 \pm \\
0.24 \mathrm{~g}\end{array}$ & $\begin{array}{l}0.77 \pm \\
0.06^{\mathrm{a}}\end{array}$ & $\begin{array}{l}1.05 \pm \\
0.00^{a}\end{array}$ & $\begin{array}{r}10.17 \pm \\
0.15^{\mathrm{f}}\end{array}$ & $\begin{array}{l}8.29 \pm \\
0.06^{\mathrm{e}}\end{array}$ & $\begin{array}{l}5.49 \pm \\
0.29^{\mathrm{d}}\end{array}$ & nd & $\begin{array}{l}1.09 \pm \\
0.15^{\mathrm{a}}\end{array}$ & $\begin{array}{l}1.60 \pm \\
0.08^{\mathrm{b}}\end{array}$ & $\begin{array}{l}1.09 \pm \\
0.02^{\mathrm{a}}\end{array}$ & $\begin{array}{l}2.12 \pm \\
0.00^{c}\end{array}$ \\
\hline Apigenin & nd & $\begin{array}{l}0.22 \pm \\
0.02^{\mathrm{a}}\end{array}$ & nd & $\begin{array}{l}0.77^{ \pm} \\
0.00^{c}\end{array}$ & $\begin{array}{l}0.91 \pm \\
0.02^{d}\end{array}$ & $\begin{array}{l}0.46 \pm \\
0.00^{\mathrm{b}}\end{array}$ & nd & $\begin{array}{l}0.78 \pm \\
0.01^{\mathrm{c}}\end{array}$ & nd & nd & nd \\
\hline Catechin & nd & $\begin{array}{c}56.75 \pm \\
3.47^{\mathrm{f}}\end{array}$ & $\begin{array}{c}69.37 \pm \\
2.40 \mathrm{~g}\end{array}$ & $\begin{array}{c}26.00 \pm \\
0.48^{\mathrm{d}}\end{array}$ & $\begin{array}{r}26.95 \pm \\
0.02 \mathrm{~d}\end{array}$ & $\begin{array}{r}22.20^{ \pm} \\
1.10^{\mathrm{c}}\end{array}$ & $\begin{array}{c}49.94 \pm \\
0.36^{\mathrm{e}}\end{array}$ & $\begin{array}{c}16.38 \pm \\
1.92^{\mathrm{a}}\end{array}$ & $\begin{array}{c}18.63 \pm \\
0.02 \mathrm{ab}\end{array}$ & $\begin{array}{c}17.24 \pm \\
0.32 \mathrm{ab}\end{array}$ & $\begin{array}{c}20.08 \pm \\
0.18^{\mathrm{bc}}\end{array}$ \\
\hline Epicatechin & $\begin{array}{c}191.69 \pm \\
2.87^{\mathrm{e}}\end{array}$ & $\begin{array}{c}267.19 \pm \\
13.52^{\mathrm{f}}\end{array}$ & $\begin{array}{l}42.32 \pm \\
1.41^{\mathrm{d}}\end{array}$ & $\begin{array}{c}21.52 \pm \\
0.4^{\mathrm{abc}}\end{array}$ & $\begin{array}{c}17.49 \pm \\
0.00^{\mathrm{ab}}\end{array}$ & $\begin{array}{c}15.79 \pm \\
0.06^{\mathrm{a}}\end{array}$ & $\begin{array}{c}29.93 \pm \\
0.29^{c}\end{array}$ & $\begin{array}{c}25.91 \pm \\
0.04 \mathrm{bc}\end{array}$ & $\begin{array}{c}17.34 \pm \\
0.86^{\mathrm{ab}}\end{array}$ & $\begin{array}{c}14.25 \pm \\
0.66^{\mathrm{a}}\end{array}$ & $\begin{array}{c}15.06 \pm \\
0.59^{\mathrm{a}}\end{array}$ \\
\hline Hesperidin & $\begin{array}{c}346.43 \pm \\
15.30^{\mathrm{d}}\end{array}$ & $\begin{array}{c}23.97 \pm \\
1.51^{\mathrm{bc}}\end{array}$ & $\begin{array}{c}3.92 \pm \\
0.25^{\mathrm{a}}\end{array}$ & $\begin{array}{r}33.89 \pm \\
0.33^{c}\end{array}$ & $\begin{array}{c}22.7 \pm \\
0.08^{\mathrm{b}}\end{array}$ & $\begin{array}{c}20.92 \pm \\
0.21^{\mathrm{b}}\end{array}$ & $\begin{array}{c}14.05 \pm \\
0.06^{\mathrm{ab}}\end{array}$ & $\begin{array}{c}3.55 \pm \\
0.12^{\mathrm{a}}\end{array}$ & $\begin{array}{c}14.75 \pm \\
0.27 \mathrm{ab}\end{array}$ & $\begin{array}{l}9.54 \pm \\
0.10^{\mathrm{a}}\end{array}$ & $\begin{array}{l}8.78 \pm \\
0.04^{\mathrm{a}}\end{array}$ \\
\hline Hispidulin & nd & $\begin{array}{l}0.93 \pm \\
0.02^{a}\end{array}$ & $\begin{array}{r}17.62^{ \pm} \\
0.75^{\mathrm{c}}\end{array}$ & $\begin{array}{l}0.73 \pm \\
0.02^{a}\end{array}$ & $\begin{array}{l}0.06 \pm \\
0.00^{\mathrm{a}}\end{array}$ & $\begin{array}{l}1.91 \pm \\
0.00^{\mathrm{b}}\end{array}$ & nd & nd & nd & nd & nd \\
\hline Isorhamnetin & $\begin{array}{r}18.75 \pm \\
0.91^{\mathrm{d}}\end{array}$ & $\begin{array}{l}4.53 \pm \\
0.21^{\mathrm{c}}\end{array}$ & $\begin{array}{l}4.45 \pm \\
0.33^{c}\end{array}$ & $\begin{array}{l}0.71 \pm \\
0.02 \mathrm{ab}\end{array}$ & $\begin{array}{l}0.68 \pm \\
0.02^{\mathrm{ab}}\end{array}$ & $\begin{array}{l}0.69 \pm \\
0.02 \mathrm{ab}\end{array}$ & $\begin{array}{l}0.96 \pm \\
0.02^{b}\end{array}$ & nd & $\begin{array}{c}0.09 \pm \\
0.00^{\mathrm{a}}\end{array}$ & $\begin{array}{l}0.06 \pm \\
0.00^{\mathrm{a}}\end{array}$ & $\begin{array}{l}0.58 \pm \\
0.04^{\mathrm{ab}}\end{array}$ \\
\hline Kaempferol & $\begin{array}{c}24.57 \pm \\
1.83^{c}\end{array}$ & $\begin{array}{c}39.56 \pm \\
0.04^{\mathrm{d}}\end{array}$ & $\begin{array}{l}1.46 \pm \\
0.08^{\mathrm{a}}\end{array}$ & $\begin{array}{l}2.21 \pm \\
0.02 \mathrm{ab}\end{array}$ & $\begin{array}{l}2.08 \pm \\
0.02 \mathrm{ab}\end{array}$ & $\begin{array}{l}1.85 \pm \\
0.04^{\mathrm{a}}\end{array}$ & $\begin{array}{l}3.20 \pm \\
0.23^{b}\end{array}$ & $\begin{array}{l}1.69 \pm \\
0.03^{\mathrm{a}}\end{array}$ & $\begin{array}{l}1.80 \pm \\
0.02^{\mathrm{a}}\end{array}$ & $\begin{array}{l}1.68 \pm \\
0.08^{\mathrm{a}}\end{array}$ & $\begin{array}{l}1.66 \pm \\
0.02^{\mathrm{a}}\end{array}$ \\
\hline Luteolin & $\begin{array}{c}15.82 \pm \\
0.79 \mathrm{e}\end{array}$ & $\begin{array}{c}5.47^{ \pm} \\
0.25^{c}\end{array}$ & $\begin{array}{r}22.77 \pm \\
0.41^{\mathrm{f}}\end{array}$ & $\begin{array}{r}12.56 \pm \\
0.08^{\mathrm{d}}\end{array}$ & $\begin{array}{l}5.53^{ \pm} \\
0.06^{c}\end{array}$ & $\begin{array}{l}3.54 \pm \\
0.02 \mathrm{ab}\end{array}$ & $\begin{array}{l}5.24 \pm \\
0.17^{c}\end{array}$ & $\begin{array}{c}5.12 \pm \\
0.13^{c}\end{array}$ & $\begin{array}{l}3.91 \pm \\
0.00 \mathrm{ab}\end{array}$ & $\begin{array}{l}4.06 \pm \\
0.02^{\mathrm{b}}\end{array}$ & $\begin{array}{l}3.28 \pm \\
0.19^{a}\end{array}$ \\
\hline Myricetin & $\begin{array}{c}20.86 \pm \\
0.24^{\mathrm{h}}\end{array}$ & $\begin{array}{c}11.13 \pm \\
0.06 \mathrm{~g}\end{array}$ & $\begin{array}{r}10.83 \pm \\
0.25^{\mathrm{f}}\end{array}$ & $\begin{array}{l}1.37 \pm \\
0.04^{\mathrm{c}}\end{array}$ & $\begin{array}{l}1.42 \pm \\
0.04^{c}\end{array}$ & $\begin{array}{c}0.68 \pm \\
0.02^{b}\end{array}$ & $\begin{array}{l}3.44 \pm \\
0.10^{\mathrm{e}}\end{array}$ & $\begin{array}{l}2.45 \pm \\
0.18^{\mathrm{d}}\end{array}$ & $\begin{array}{l}1.27 \pm \\
0.08^{c}\end{array}$ & $\begin{array}{c}0.22 \pm \\
0.02^{\mathrm{a}}\end{array}$ & $\begin{array}{c}0.25 \pm \\
0.02^{a}\end{array}$ \\
\hline Naringin & $\begin{array}{c}523.85 \pm \\
23.71 \text { ef }\end{array}$ & $\begin{array}{c}691.17 \pm \\
2.68^{\mathrm{h}}\end{array}$ & $\begin{array}{c}683.18 \pm \\
47.10^{\mathrm{h}}\end{array}$ & $\begin{array}{c}550.60 \pm \\
1.22^{\mathrm{f}}\end{array}$ & $\begin{array}{c}402.84 \pm \\
0.41^{\mathrm{cd}}\end{array}$ & $\begin{array}{c}440.54 \pm \\
0.47^{\mathrm{d}}\end{array}$ & $\begin{array}{c}596.81 \pm \\
25.89 \mathrm{~g}\end{array}$ & $\begin{array}{c}304.06 \pm \\
2.51^{\mathrm{a}}\end{array}$ & $\begin{array}{c}494.46 \pm \\
1.00 \mathrm{e}^{\mathrm{e}}\end{array}$ & $\begin{array}{c}350.50 \pm \\
0.95^{\mathrm{b}}\end{array}$ & $\begin{array}{c}396.56 \pm \\
10.59^{\mathrm{c}}\end{array}$ \\
\hline Quercetin & $\begin{array}{l}9.74 \pm \\
0.12 \mathrm{~g}\end{array}$ & $\begin{array}{c}10.92 \pm \\
0.23^{\mathrm{h}}\end{array}$ & $\begin{array}{c}10.95 \pm \\
0.58^{\mathrm{h}}\end{array}$ & $\begin{array}{l}8.70 \pm \\
0.17^{\mathrm{f}}\end{array}$ & $\begin{array}{c}7.95 \pm \\
0.37^{\mathrm{e}}\end{array}$ & $\begin{array}{l}7.09 \pm \\
0.18^{d}\end{array}$ & $\begin{array}{l}9.18 \pm \\
0.37 \mathrm{fg}\end{array}$ & $\begin{array}{l}5.41 \pm \\
0.09^{c}\end{array}$ & $\begin{array}{l}4.21 \pm \\
0.06^{\mathrm{b}}\end{array}$ & $\begin{array}{l}5.49 \pm \\
0.02^{c}\end{array}$ & $\begin{array}{l}3.37 \pm \\
0.23^{a}\end{array}$ \\
\hline Rutin & $\begin{array}{c}317.77 \pm \\
7.68^{\mathrm{h}}\end{array}$ & $\begin{array}{c}27.01 \mathrm{ef} \\
0.69 \mathrm{ef}\end{array}$ & $\begin{array}{c}54.32 \mathrm{I} \\
4.80^{\mathrm{g}}\end{array}$ & $\begin{array}{l}20.54 \pm \\
0.19^{\text {cde }}\end{array}$ & $\begin{array}{c}17.55 \pm \\
0.13^{\mathrm{cd}}\end{array}$ & $\begin{array}{l}7.78 \pm \\
0.65^{\mathrm{ab}}\end{array}$ & $\begin{array}{c}24.40 \pm \\
1.22 \mathrm{de}\end{array}$ & $\begin{array}{l}33.30 \pm \\
7.21^{\mathrm{f}}\end{array}$ & $\begin{array}{c}15.22 \pm \\
0.08^{\mathrm{bc}}\end{array}$ & $\begin{array}{l}6.23 \pm \\
0.10^{a}\end{array}$ & $\begin{array}{l}3.78 \pm \\
0.08^{a}\end{array}$ \\
\hline \multicolumn{12}{|l|}{$\begin{array}{l}\text { Phenolic } \\
\text { diterpenes: }\end{array}$} \\
\hline Carnosic acid & $\begin{array}{l}22.63 \pm \\
1.77^{\mathrm{abc}}\end{array}$ & $\begin{array}{c}235.33 \pm \\
1.40 \mathrm{gh}\end{array}$ & $\begin{array}{c}249.48 \pm \\
13.41^{\mathrm{h}}\end{array}$ & $\begin{array}{c}225.95 \pm \\
14.83 \mathrm{~g}\end{array}$ & $\begin{array}{c}196.02 \pm \\
1.60^{\mathrm{f}}\end{array}$ & $\begin{array}{c}132.17 \pm \\
5.08 \mathrm{e}^{ \pm}\end{array}$ & $\begin{array}{l}36.51 \pm \\
3.25^{\mathrm{c}}\end{array}$ & $\begin{array}{c}98.80 \pm \\
5.33^{\mathrm{d}}\end{array}$ & $\begin{array}{c}26.63 \pm \\
0.20^{\mathrm{bc}}\end{array}$ & $\begin{array}{c}14.54 \pm \\
0.50 \mathrm{ab}\end{array}$ & $\begin{array}{c}10.52 \pm \\
0.44^{\mathrm{a}}\end{array}$ \\
\hline Carnosol & $\begin{array}{c}151.86 \pm \\
1.77^{\mathrm{e}}\end{array}$ & $\begin{array}{c}381.79 \pm \\
0.02^{\mathrm{h}}\end{array}$ & $\begin{array}{c}133.87 \pm \\
7.20^{\mathrm{d}}\end{array}$ & $\begin{array}{c}273.13 \pm \\
0.02^{\mathrm{f}}\end{array}$ & $\begin{array}{c}302.41 \pm \\
0.37 \mathrm{~g}\end{array}$ & $\begin{array}{c}275.11 \pm \\
0.29^{\mathrm{f}}\end{array}$ & $\begin{array}{c}27.84 \pm \\
0.08^{\mathrm{a}}\end{array}$ & $\begin{array}{c}120.38 \pm \\
0.21^{\mathrm{c}}\end{array}$ & $\begin{array}{c}39.20 \pm \\
2.15^{\mathrm{b}}\end{array}$ & $\begin{array}{c}40.71 \pm \\
0.15^{\mathrm{b}}\end{array}$ & $\begin{array}{c}37.80 \pm \\
0.77^{\mathrm{b}}\end{array}$ \\
\hline
\end{tabular}

Results are expressed as mean \pm SD $(n=3)$. DW-dry weight; nd-not determined. Mean values with different letters $(\mathrm{a}-\mathrm{i})$ within the individual rows are statistically different $(p<0.05)$.

In all samples, storage resulted in a decrease in the content of particular polyphenolic compounds. However, the rates of loss during storage were different for individual compounds. 
Table 7. Concentration of individual polyphenolic compounds in fresh, dried, and stored Salvia sclarea.

\begin{tabular}{|c|c|c|c|c|c|c|c|c|c|c|c|}
\hline \multirow{3}{*}{$\begin{array}{l}\text { Polyphenolic } \\
\text { Compounds }\end{array}$} & \multirow[b]{2}{*}{ Fresh } & \multicolumn{5}{|c|}{ Directly after Drying } & \multicolumn{5}{|c|}{ After 12 Months of Storage } \\
\hline & & $\begin{array}{l}\text { Freeze- } \\
\text { Drying }\end{array}$ & $\begin{array}{l}\text { Natural } \\
\text { Drying }\end{array}$ & $\begin{array}{l}\text { Drying } \\
\text { at } 30^{\circ} \mathrm{C}\end{array}$ & $\begin{array}{l}\text { Drying } \\
\text { at } 40^{\circ} \mathrm{C}\end{array}$ & $\begin{array}{l}\text { Drying } \\
\text { at } 50^{\circ} \mathrm{C}\end{array}$ & $\begin{array}{l}\text { Freeze- } \\
\text { Drying }\end{array}$ & $\begin{array}{l}\text { Natural } \\
\text { Drying }\end{array}$ & $\begin{array}{l}\text { Drying } \\
\text { at } 30^{\circ} \mathrm{C}\end{array}$ & $\begin{array}{l}\text { Drying } \\
\text { at } 40^{\circ} \mathrm{C}\end{array}$ & $\begin{array}{l}\text { Drying } \\
\text { at } 50^{\circ} \mathrm{C}\end{array}$ \\
\hline & \multicolumn{11}{|c|}{$\mathrm{mg} \cdot 100 \mathrm{~g}^{-1} \mathrm{DW}$} \\
\hline \\
\hline $\begin{array}{c}\text { Acids } \\
4- \\
\text { Hydroxybenzoic }\end{array}$ & $\begin{array}{c}57.28 \pm \\
0.17^{\mathrm{k}}\end{array}$ & $\begin{array}{r}20.11 \pm \\
0.12^{j}\end{array}$ & $\begin{array}{r}18.09 \pm \\
0.00^{\mathrm{i}}\end{array}$ & $\begin{array}{l}6.59 \pm \\
0.11^{\mathrm{e}}\end{array}$ & $\begin{array}{l}6.26 \pm \\
0.02^{d}\end{array}$ & $\begin{array}{l}9.73 \pm \\
0.04^{h}\end{array}$ & $\begin{array}{l}7.74 \pm \\
0.04^{\mathrm{f}}\end{array}$ & $\begin{array}{l}5.53 \pm \\
0.00^{c}\end{array}$ & $\begin{array}{l}5.09 \pm \\
0.04^{\mathrm{a}}\end{array}$ & $\begin{array}{l}5.28 \pm \\
0.00^{b}\end{array}$ & $\begin{array}{l}8.03 \pm \\
0.02 \mathrm{~g}\end{array}$ \\
\hline Caffeic acid & $\begin{array}{c}31.94 \pm \\
0.00^{\mathrm{i}}\end{array}$ & $\begin{array}{c}42.61 \pm \\
0.00^{\mathrm{k}}\end{array}$ & $\begin{array}{c}15.47 \pm \\
0.00 \mathrm{e}^{\mathrm{e}}\end{array}$ & $\begin{array}{r}17.48 \pm \\
0.04^{\mathrm{g}}\end{array}$ & $\begin{array}{c}19.57 \pm \\
0.02^{\mathrm{h}}\end{array}$ & $\begin{array}{c}33.61 \pm \\
0.02^{j}\end{array}$ & $\begin{array}{r}15.04 \pm \\
0.00 \mathrm{~d}\end{array}$ & $\begin{array}{l}8.94 \pm \\
0.03^{\mathrm{a}}\end{array}$ & $\begin{array}{r}12.51 \pm \\
0.00^{\mathrm{c}}\end{array}$ & $\begin{array}{c}11.93 \pm \\
0.00^{\mathrm{b}}\end{array}$ & $\begin{array}{r}15.62 \pm \\
0.10^{\mathrm{f}}\end{array}$ \\
\hline $\begin{array}{l}\text { Chlorogenic } \\
\text { acid }\end{array}$ & $\begin{array}{c}70.61 \pm \\
0.34^{\mathrm{i}}\end{array}$ & $\begin{array}{c}54.28 \pm \\
0.08^{\mathrm{h}}\end{array}$ & $\begin{array}{c}34.98 \pm \\
0.17^{\mathrm{f}}\end{array}$ & $\begin{array}{r}27.12 \mathrm{I} \\
0.23^{\mathrm{d}}\end{array}$ & $\begin{array}{c}34.38 \pm \\
0.04 \mathrm{e}^{\mathrm{e}}\end{array}$ & $\begin{array}{c}35.54 \pm \\
0.02 \mathrm{~g}\end{array}$ & $\begin{array}{c}35.49 \pm \\
0.04 \mathrm{~g}\end{array}$ & $\begin{array}{r}19.89^{ \pm} \\
0.04^{\mathrm{c}}\end{array}$ & $\begin{array}{r}27.11 \pm \\
0.02^{\mathrm{d}}\end{array}$ & $\begin{array}{l}4.71 \pm \\
0.19^{b}\end{array}$ & $\begin{array}{l}3.87 \pm \\
0.08^{\mathrm{a}}\end{array}$ \\
\hline Ferulic acid & $\begin{array}{c}7.57 \pm \\
0.51^{\mathrm{a}}\end{array}$ & $\begin{array}{l}17.28 \pm \\
0.76^{\mathrm{f}}\end{array}$ & $\begin{array}{c}27.55 \pm \\
0.25^{\mathrm{h}}\end{array}$ & $\begin{array}{r}20.36 \pm \\
0.02 \mathrm{~g}\end{array}$ & $\begin{array}{r}20.20 \pm \\
0.48^{\mathrm{g}}\end{array}$ & $\begin{array}{r}13.70 \pm \\
0.00^{\mathrm{c}}\end{array}$ & $\begin{array}{r}13.77 \pm \\
0.00^{\mathrm{c}}\end{array}$ & $\begin{array}{l}8.79 \pm \\
0.14^{\mathrm{b}}\end{array}$ & $\begin{array}{c}15.94 \pm \\
0.08^{\mathrm{e}}\end{array}$ & $\begin{array}{r}15.10 \pm \\
0.04^{\mathrm{d}}\end{array}$ & $\begin{array}{l}8.54 \pm \\
0.00^{\mathrm{b}}\end{array}$ \\
\hline Gallic acid & $\begin{array}{c}40.71 \pm \\
0.51^{\mathrm{a}}\end{array}$ & $\begin{array}{c}699.97 \pm \\
0.10^{\mathrm{k}}\end{array}$ & $\begin{array}{c}293.84 \pm \\
0.25^{c}\end{array}$ & $\begin{array}{c}453.90 \pm \\
0.29 \mathrm{~g}\end{array}$ & $\begin{array}{c}421.89 \pm \\
0.27^{\mathrm{e}}\end{array}$ & $\begin{array}{c}604.09 \pm \\
0.311^{\mathrm{i}}\end{array}$ & $\begin{array}{c}648.01 \pm \\
0.08^{\mathrm{j}}\end{array}$ & $\begin{array}{c}252.22 \pm \\
0.58^{\mathrm{b}}\end{array}$ & $\begin{array}{c}424.33 \pm \\
0.02^{\mathrm{f}}\end{array}$ & $\begin{array}{c}416.35 \pm \\
0.48^{\mathrm{d}}\end{array}$ & $\begin{array}{c}563.42 \pm \\
2.65^{\mathrm{h}}\end{array}$ \\
\hline $\begin{array}{l}\text { p-Coumaric } \\
\text { acid }\end{array}$ & nd & $\begin{array}{l}2.09 \pm \\
0.02^{d}\end{array}$ & nd & $\begin{array}{l}1.86 \pm \\
0.02^{\mathrm{b}}\end{array}$ & $\begin{array}{c}2.16 \pm \\
0.10^{\mathrm{d}}\end{array}$ & $\begin{array}{l}2.07 \pm \\
0.00 \mathrm{~cd}\end{array}$ & $\begin{array}{l}1.36 \pm \\
0.02^{a}\end{array}$ & nd & $\begin{array}{l}1.94 \pm \\
0.02^{\mathrm{bc}}\end{array}$ & $\begin{array}{l}1.91 \pm \\
0.02^{b}\end{array}$ & $\begin{array}{l}1.95 \pm \\
0.10^{\mathrm{bc}}\end{array}$ \\
\hline $\begin{array}{l}\text { Rosmarinic } \\
\text { acid }\end{array}$ & $\begin{array}{c}346.32 \pm \\
1.02^{\mathrm{a}}\end{array}$ & $\begin{array}{c}2167.94 \pm \\
0.23^{\mathrm{i}}\end{array}$ & $\begin{array}{c}1839.50 \pm \\
0.59 \mathrm{~g}\end{array}$ & $\begin{array}{c}1730.68 \pm \\
0.33 \mathrm{~g}\end{array}$ & $\begin{array}{c}1891.31 \pm \\
1.66^{\mathrm{h}}\end{array}$ & $\begin{array}{c}2368.20 \pm \\
1.49^{\mathrm{j}}\end{array}$ & $\begin{array}{c}382.38 \pm \\
0.41^{\mathrm{b}}\end{array}$ & $\begin{array}{c}1101.51 \pm \\
0.10^{\mathrm{c}}\end{array}$ & $\begin{array}{c}1108.78 \pm \\
0.76^{\mathrm{d}}\end{array}$ & $\begin{array}{c}1203.75 \pm \\
0.04 \mathrm{e}^{\mathrm{e}}\end{array}$ & $\begin{array}{c}1296.03 \pm \\
0.64^{\mathrm{f}}\end{array}$ \\
\hline Sinapinic acid & $\begin{array}{r}20.65 \pm \\
0.34 \mathrm{~d}\end{array}$ & $\begin{array}{c}25.81 \pm \\
0.49^{\mathrm{e}}\end{array}$ & $\begin{array}{c}10.23 \pm \\
0.17^{\mathrm{b}}\end{array}$ & $\begin{array}{r}18.09 \pm \\
1.90^{\mathrm{c}}\end{array}$ & $\begin{array}{c}20.93 \pm \\
0.04^{\mathrm{d}}\end{array}$ & $\begin{array}{c}29.88 \pm \\
0.04^{\mathrm{f}}\end{array}$ & $\begin{array}{l}3.74 \pm \\
0.00^{\mathrm{a}}\end{array}$ & $\begin{array}{l}9.41 \pm \\
0.46^{\mathrm{b}}\end{array}$ & $\begin{array}{c}3.33 \pm \\
0.00^{\mathrm{a}}\end{array}$ & $\begin{array}{c}3.24 \pm \\
0.10^{\mathrm{a}}\end{array}$ & $\begin{array}{r}18.13 \pm \\
0.04^{\mathrm{c}}\end{array}$ \\
\hline Syringic acid & $\begin{array}{c}7.81 \pm \\
0.17^{\mathrm{a}}\end{array}$ & $\begin{array}{r}29.07 \pm \\
0.02^{\mathrm{h}}\end{array}$ & $\begin{array}{c}63.36 \pm \\
0.08^{\mathrm{i}}\end{array}$ & $\begin{array}{c}24.51 \pm \\
0.02 \mathrm{~g}\end{array}$ & $\begin{array}{c}23.6 \pm \\
0.00^{\mathrm{f}}\end{array}$ & $\begin{array}{c}29.31 \pm \\
0.00^{\mathrm{h}}\end{array}$ & $\begin{array}{r}18.15 \pm \\
0.04^{\mathrm{c}}\end{array}$ & $\begin{array}{c}13.66 \pm \\
0.01^{\mathrm{b}}\end{array}$ & $\begin{array}{r}19.49 \pm \\
0.02\end{array}$ & $\begin{array}{c}19.85 \pm \\
0.33^{\mathrm{e}}\end{array}$ & $\begin{array}{r}24.43 \pm \\
0.02 \mathrm{~g}\end{array}$ \\
\hline Vanillic acid & $\begin{array}{r}11.29 \pm \\
0.34^{\mathrm{g}}\end{array}$ & $\begin{array}{l}2.62 \pm \\
0.06^{\text {cde }}\end{array}$ & $\begin{array}{c}3.81 \pm \\
0.00^{\mathrm{f}}\end{array}$ & $\begin{array}{l}2.70 \pm \\
0.06^{\mathrm{de}}\end{array}$ & $\begin{array}{l}2.82 \pm \\
0.04 \\
\end{array}$ & $\begin{array}{l}2.51 \pm \\
0.04^{\mathrm{cd}}\end{array}$ & $\begin{array}{l}2.51 \pm \\
0.04^{\mathrm{cd}}\end{array}$ & $\begin{array}{l}1.59 \pm \\
0.08^{\mathrm{a}}\end{array}$ & $\begin{array}{l}2.42 \pm \\
0.02 \mathrm{bc}\end{array}$ & $\begin{array}{l}2.40 \pm \\
0.04^{\mathrm{bc}}\end{array}$ & $\begin{array}{l}2.17 \pm \\
0.02^{b}\end{array}$ \\
\hline \multicolumn{12}{|l|}{ Flavonoids } \\
\hline Acacetin & nd & $\begin{array}{c}11.59 \pm \\
0.04 \mathrm{~g}\end{array}$ & $\begin{array}{l}3.81 \pm \\
0.00^{\mathrm{e}}\end{array}$ & $\begin{array}{l}2.25 \pm \\
0.00^{c}\end{array}$ & $\begin{array}{l}2.84 \pm \\
0.02^{d}\end{array}$ & $\begin{array}{l}1.13 \pm \\
0.04^{\mathrm{b}}\end{array}$ & $\begin{array}{l}6.83 \pm \\
0.02^{\mathrm{f}}\end{array}$ & $\begin{array}{l}0.76 \pm \\
0.01^{\mathrm{a}}\end{array}$ & nd & nd & nd \\
\hline Apigenin & $\begin{array}{l}6.72^{ \pm} \\
0.34^{c}\end{array}$ & nd & $\begin{array}{l}3.33 \pm \\
0.17^{b}\end{array}$ & nd & nd & nd & nd & $\begin{array}{l}0.45 \pm \\
0.03^{\mathrm{a}}\end{array}$ & nd & nd & nd \\
\hline Epicatechin & $\begin{array}{c}89.34 \pm \\
0.34^{\mathrm{d}}\end{array}$ & $\begin{array}{c}131.06 \pm \\
0.04 \mathrm{~g}\end{array}$ & $\begin{array}{c}128.69 \pm \\
0.25^{\mathrm{f}}\end{array}$ & $\begin{array}{c}97.95 \pm \\
0.31 \mathrm{e}\end{array}$ & $\begin{array}{c}145.49 \pm \\
0.27^{\mathrm{h}}\end{array}$ & $\begin{array}{c}211.60 \pm \\
0.31 \mathrm{j}\end{array}$ & $\begin{array}{c}87.21 \pm \\
0.60^{c}\end{array}$ & $\begin{array}{c}86.06 \pm \\
0.07^{\mathrm{b}}\end{array}$ & $\begin{array}{c}15.65 \pm \\
0.02^{\mathrm{a}}\end{array}$ & $\begin{array}{c}15.01 \pm \\
0.17^{\mathrm{a}}\end{array}$ & $\begin{array}{c}180.71 \pm \\
0.55^{\mathrm{i}}\end{array}$ \\
\hline Hesperidin & $\begin{array}{c}33.02 \pm \\
0.17^{\mathrm{g}}\end{array}$ & $\begin{array}{c}122.87 \\
0.08^{\mathrm{i}}\end{array}$ & $\begin{array}{c}131.6 \pm \\
0.17^{\mathrm{j}}\end{array}$ & $\begin{array}{c}161.50 \pm \\
0.08^{\mathrm{k}}\end{array}$ & $\begin{array}{r}11.28 \pm \\
0.08^{\mathrm{d}}\end{array}$ & $\begin{array}{c}85.56 \pm \\
0.04^{\mathrm{h}}\end{array}$ & $\begin{array}{c}7.95 \pm \\
0.10^{\mathrm{a}}\end{array}$ & $\begin{array}{r}25.76 \pm \\
0.01^{\mathrm{f}}\end{array}$ & $\begin{array}{c}18.57 \pm \\
0.11^{\mathrm{e}}\end{array}$ & $\begin{array}{l}8.43 \pm \\
0.06^{\mathrm{b}}\end{array}$ & $\begin{array}{r}10.83 \pm \\
0.10^{\mathrm{c}}\end{array}$ \\
\hline Isorhamnetin & $\begin{array}{c}61.48 \pm \\
1.70^{\mathrm{e}}\end{array}$ & $\begin{array}{l}4.36 \pm \\
0.04 \stackrel{\mathrm{bc}}{ }\end{array}$ & $\begin{array}{r}30.46 \pm \\
0.84^{\mathrm{d}}\end{array}$ & $\begin{array}{l}3.67 \pm \\
0.02^{\mathrm{b}}\end{array}$ & $\begin{array}{l}4.11 \pm \\
0.02^{b}\end{array}$ & $\begin{array}{l}3.92 \pm \\
0.08^{b}\end{array}$ & $\begin{array}{l}3.34 \pm \\
0.02^{b}\end{array}$ & $\begin{array}{l}5.43 \pm \\
0.10^{c}\end{array}$ & $\begin{array}{c}0.39 \pm \\
0.04^{\mathrm{a}}\end{array}$ & $\begin{array}{l}0.62 \pm \\
0.00^{\mathrm{a}}\end{array}$ & $\begin{array}{c}0.55 \pm \\
0.04^{\mathrm{a}}\end{array}$ \\
\hline Kaempferol & nd & $\begin{array}{l}9.33 \pm \\
0.04^{\mathrm{f}}\end{array}$ & nd & $\begin{array}{l}2.71 \pm \\
0.04^{\mathrm{a}}\end{array}$ & $\begin{array}{l}4.19 \pm \\
0.04^{\mathrm{d}}\end{array}$ & $\begin{array}{l}8.35 \pm \\
0.02 \mathrm{e}\end{array}$ & $\begin{array}{l}3.78 \pm \\
0.02^{\mathrm{c}}\end{array}$ & nd & $\begin{array}{l}3.24 \pm \\
0.04^{b}\end{array}$ & nd & nd \\
\hline Luteolin & $\begin{array}{l}31.7 \pm \\
0.34 \mathrm{~g}\end{array}$ & $\begin{array}{l}5.41 \pm \\
0.02 \mathrm{de}\end{array}$ & $\begin{array}{c}17.67 \pm \\
1.26^{\mathrm{f}}\end{array}$ & $\begin{array}{l}4.62 \mathrm{t} \\
0.29^{\mathrm{cd}}\end{array}$ & $\begin{array}{l}3.71 \pm \\
0.08 \mathrm{bc}\end{array}$ & $\begin{array}{l}5.81 \pm \\
0.06^{\mathrm{e}}\end{array}$ & $\begin{array}{l}4.08 \pm \\
0.02 \mathrm{bc}\end{array}$ & $\begin{array}{l}4.47 \underset{ \pm}{ \pm} \\
0.14^{\mathrm{cd}}\end{array}$ & $\begin{array}{l}2.72 \pm \\
0.15^{\mathrm{a}}\end{array}$ & $\begin{array}{l}2.29 \pm \\
0.08^{a}\end{array}$ & $\begin{array}{l}3.15 \pm \\
0.12 \mathrm{ab}\end{array}$ \\
\hline Myricetin & nd & $\begin{array}{c}31.33 \pm \\
0.10^{\mathrm{e}}\end{array}$ & $\begin{array}{c}11.66 \pm \\
0.17^{\mathrm{b}}\end{array}$ & $\begin{array}{c}37.63 \pm \\
0.02^{\mathrm{h}}\end{array}$ & $\begin{array}{c}36.22 \pm \\
0.25 \mathrm{~g}\end{array}$ & $\begin{array}{r}12.25 \pm \\
0.02^{\mathrm{c}}\end{array}$ & $\begin{array}{r}28.46 \pm \\
0.04\end{array}$ & $\begin{array}{c}1.22 \pm \\
0.09^{\mathrm{a}}\end{array}$ & $\begin{array}{r}32.32 \pm \\
0.00^{\mathrm{f}}\end{array}$ & nd & nd \\
\hline Naringin & $\begin{array}{c}30.26 \pm \\
1.70^{\mathrm{a}}\end{array}$ & $\begin{array}{c}186.74 \pm \\
0.19^{\mathrm{f}}\end{array}$ & $\begin{array}{c}252.37 \\
1.68^{\mathrm{i}} \pm\end{array}$ & $\begin{array}{c}271.53 \pm \\
1.53^{\mathrm{j}}\end{array}$ & $\begin{array}{c}300.27 \pm \\
0.17^{\mathrm{k}}\end{array}$ & $\begin{array}{c}128.11 \pm \\
0.08^{\mathrm{d}}\end{array}$ & $\begin{array}{c}148.44 \pm \\
0.31 \mathrm{e}^{\mathrm{e}}\end{array}$ & $\begin{array}{c}108.01 \mathrm{t} \\
0.15^{\mathrm{b}}\end{array}$ & $\begin{array}{c}247.12 \pm \\
0.14^{\mathrm{h}}\end{array}$ & $\begin{array}{c}237.02 \pm \\
0.19 \mathrm{~g}\end{array}$ & $\begin{array}{c}120.52 \pm \\
0.04^{\mathrm{c}}\end{array}$ \\
\hline Quercetin & nd & $\begin{array}{c}3.12 \pm \\
0.19^{c}\end{array}$ & nd & $\begin{array}{c}5.33 \pm \\
0.144^{\mathrm{e}}\end{array}$ & $\begin{array}{c}6.13 \pm \\
0.06^{\mathrm{f}}\end{array}$ & $\begin{array}{c}3.54 \pm \\
0.14^{\mathrm{d}}\end{array}$ & $\begin{array}{l}1.36 \pm \\
0.02^{\mathrm{a}}\end{array}$ & nd & $\begin{array}{c}2.90^{ \pm} \\
0.15^{\mathrm{c}}\end{array}$ & $\begin{array}{l}3.43 \pm \\
0.08^{\mathrm{d}}\end{array}$ & $\begin{array}{l}1.98 \pm \\
0.02^{\mathrm{b}}\end{array}$ \\
\hline Rutin & $\begin{array}{c}127.41 \pm \\
0.85^{\mathrm{e}}\end{array}$ & $\begin{array}{c}207.67 \pm \\
0.11 \mathrm{~g}\end{array}$ & $\begin{array}{c}52.77 \pm \\
2.27 \mathrm{~d}\end{array}$ & $\begin{array}{c}236.58 \pm \\
0.04^{\mathrm{h}}\end{array}$ & $\begin{array}{l}8.55 \pm \\
0.02^{b}\end{array}$ & $\begin{array}{c}146.97 \pm \\
0.04^{\mathrm{f}}\end{array}$ & $\begin{array}{l}7.46 \pm \\
0.14^{\mathrm{b}}\end{array}$ & $\begin{array}{c}46.17 \pm \\
0.17^{\mathrm{c}}\end{array}$ & $\begin{array}{l}5.23 \pm \\
0.13^{\mathrm{b}}\end{array}$ & $\begin{array}{l}4.88 \pm \\
0.06^{\mathrm{a}}\end{array}$ & $\begin{array}{l}8.04 \pm \\
0.16^{\mathrm{b}}\end{array}$ \\
\hline \multicolumn{12}{|l|}{$\begin{array}{l}\text { Phenolic } \\
\text { diterpenes }\end{array}$} \\
\hline Carnosol & $\begin{array}{c}296.72 \pm \\
10.70^{\mathrm{f}}\end{array}$ & $\begin{array}{c}108.71 \pm \\
4.33 \mathrm{de}\end{array}$ & $\begin{array}{c}76.03 \pm \\
0.67^{\mathrm{b}}\end{array}$ & $\begin{array}{c}91.84 \pm \\
0.00^{\mathrm{c}}\end{array}$ & $\begin{array}{c}116.73 \pm \\
0.23 \mathrm{e}^{ \pm}\end{array}$ & $\begin{array}{c}115.40 \pm \\
6.26 \mathrm{de}\end{array}$ & $\begin{array}{c}93.59 \pm \\
0.27^{\mathrm{c}}\end{array}$ & $\begin{array}{c}65.92 \pm \\
4.48^{\mathrm{a}}\end{array}$ & $\begin{array}{c}73.74 \pm \\
0.25^{\mathrm{ab}}\end{array}$ & $\begin{array}{c}107.78 \pm \\
0.23 \mathrm{de}\end{array}$ & $\begin{array}{c}106.56 \pm \\
0.08^{\mathrm{d}}\end{array}$ \\
\hline Carnosic acid & $\begin{array}{r}53.44 \pm \\
0.51^{\mathrm{f}}\end{array}$ & $\begin{array}{c}113.04 \pm \\
5.55^{\mathrm{h}}\end{array}$ & $\begin{array}{r}56.46 \pm \\
2.94^{\mathrm{f}}\end{array}$ & $\begin{array}{c}76.75 \pm \\
2.08 \mathrm{~g}\end{array}$ & $\begin{array}{c}46.33 \pm \\
2.07^{\mathrm{e}}\end{array}$ & $\begin{array}{c}35.25 \pm \\
1.42^{\mathrm{d}}\end{array}$ & $\begin{array}{c}35.55 \pm \\
2.37^{\mathrm{d}}\end{array}$ & $\begin{array}{c}1.53 \pm \\
0.10^{\mathrm{a}}\end{array}$ & $\begin{array}{l}9.16 \pm \\
0.48^{\mathrm{bc}}\end{array}$ & $\begin{array}{c}6.69 \pm \\
0.44^{\mathrm{b}}\end{array}$ & $\begin{array}{r}13.99 \pm \\
0.57^{\mathrm{c}}\end{array}$ \\
\hline
\end{tabular}

Results are expressed as mean \pm SD $(n=3)$. DW-dry weight; nd-not determined. Mean values with different letters $(\mathrm{a}-\mathrm{k})$ within the individual rows are statistically different $(p<0.05)$.

\subsection{Antioxidant Activity}

The results indicated that the fresh herbs Salvia hispanica and Salvia officinalis were characterized by higher antioxidant activity $\left(713.26 \mu \mathrm{mol}\right.$ Trolox $\cdot \mathrm{g}^{-1} \mathrm{DW}$ and $651.48 \mu \mathrm{mol}$ Trolox $\cdot \mathrm{g}^{-1} \mathrm{DW}$, respectively) than Salvia sclarea $\left(568.49 \mu \mathrm{mol}\right.$ Trolox $\left.\cdot \mathrm{g}^{-1} \mathrm{DW}\right)$ (Table 2). In the herbs Salvia hispanica and Salvia officinalis after freeze-drying, natural drying, and drying at $30{ }^{\circ} \mathrm{C}$ and $40^{\circ} \mathrm{C}$, an increase in the ability to scavenge free radicals (highest in the samples after freeze-drying and natural drying) was observed (Table 8). In these groups of samples, drying at $50{ }^{\circ} \mathrm{C}$ caused a decrease in the antioxidant activity. In the herb Salvia sclarea the highest increase in antioxidant capacity was found in the sample after natural drying, and the lowest was measured after drying at $50^{\circ} \mathrm{C}$. After 3,6 , and 12 months of storage, a decrease in the ability to scavenge free radicals was observed. 
Table 8. The antioxidant activity in dried and stored sage of individual species.

\begin{tabular}{|c|c|c|c|c|c|c|c|c|c|}
\hline \multirow[b]{3}{*}{ Species } & \multirow[b]{3}{*}{$\begin{array}{l}\text { Drying } \\
\text { Method }\end{array}$} & \multicolumn{8}{|c|}{ Storage } \\
\hline & & \multicolumn{2}{|c|}{ Directly after Drying } & \multicolumn{2}{|c|}{ After 3 Months } & \multicolumn{2}{|c|}{ After 6 Months } & \multicolumn{2}{|c|}{ After 12 Months } \\
\hline & & $\begin{array}{l}\text { Antioxidant } \\
\text { Capacity } \\
\text { [ } \mu \mathrm{mol} \\
\text { Trolox·g } \\
\text { DW] }\end{array}$ & $\begin{array}{c}\text { Changes * } \\
{[\%]}\end{array}$ & 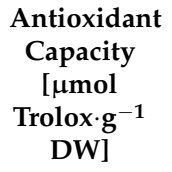 & $\begin{array}{c}\text { Changes } \\
{[\%]}\end{array}$ & 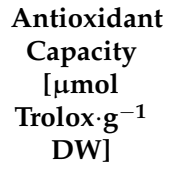 & $\begin{array}{c}\text { Changes ** } \\
{[\%]}\end{array}$ & 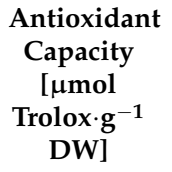 & $\begin{array}{c}\text { Changes ** } \\
{[\%]}\end{array}$ \\
\hline \multirow{5}{*}{$\begin{array}{c}\text { Salvia } \\
\text { hispanica }\end{array}$} & $\begin{array}{l}\text { freeze- } \\
\text { drying }\end{array}$ & $\begin{array}{l}1069.05 \pm \\
33.52^{\mathrm{h}, \mathrm{C}}\end{array}$ & 49.88 & $\begin{array}{l}869.48 \pm \\
34.27 \mathrm{hi,} \mathrm{B}\end{array}$ & -18.67 & $\begin{array}{l}639.10 \pm \\
10.29 \mathrm{~h}, \mathrm{~A}\end{array}$ & -40.22 & $\begin{array}{l}621.54 \pm \\
21.09 \mathrm{~g}, \mathrm{~A}\end{array}$ & -41.86 \\
\hline & $\begin{array}{l}\text { natural } \\
\text { drying }\end{array}$ & $\begin{array}{l}1074.27 \pm \\
10.56^{\text {h, D }}\end{array}$ & 50.61 & $\begin{array}{l}897.15 \pm \\
10.33^{i, C}\end{array}$ & -16.49 & $\begin{array}{c}681.72 \pm \\
5.16^{\mathrm{i}, \mathrm{B}}\end{array}$ & -36.54 & $\begin{array}{l}614.79 \pm \\
10.49 \mathrm{~g}, \mathrm{~A}\end{array}$ & -42.77 \\
\hline & $\begin{array}{l}\text { drying at } \\
30^{\circ} \mathrm{C}\end{array}$ & $\begin{array}{l}840.23 \pm \\
10.67 \mathrm{e}, \mathrm{B}\end{array}$ & 17.80 & $\begin{array}{c}811.02 \pm \\
7.92 \mathrm{~h}, \mathrm{~B}\end{array}$ & -3.48 & $\begin{array}{l}520.08 \pm \\
18.23^{f, A}\end{array}$ & -38.10 & $\begin{array}{c}506.33 \pm \\
8.00^{\mathrm{f}, \mathrm{A}}\end{array}$ & -39.74 \\
\hline & $\begin{array}{l}\text { drying at } \\
40^{\circ} \mathrm{C}\end{array}$ & $\begin{array}{l}849.28 \pm \\
10.32 \mathrm{ef}, \mathrm{C}\end{array}$ & 19.07 & $\begin{array}{l}816.21 \pm \\
44.56 \mathrm{~h}, \mathrm{C}\end{array}$ & -3.89 & $\begin{array}{l}522.63 \pm \\
12.90^{f, B}\end{array}$ & -38.46 & $\begin{array}{c}420.40 \pm \\
2.65^{\mathrm{e}, \mathrm{A}}\end{array}$ & -50.50 \\
\hline & $\begin{array}{c}\text { drying at } \\
50^{\circ} \mathrm{C}\end{array}$ & $\begin{array}{l}507.94 \pm \\
15.76^{a}, C\end{array}$ & -28.79 & $\begin{array}{c}361.70 \pm \\
2.60^{a}, \mathrm{~B}\end{array}$ & -28.79 & $\begin{array}{c}125.97 \pm \\
2.60^{\mathrm{a}}, \mathrm{A}\end{array}$ & -75.20 & $\begin{array}{c}118.31 \pm \\
7.79 \mathrm{a}, \mathrm{A}\end{array}$ & -76.71 \\
\hline \multirow{5}{*}{$\begin{array}{l}\text { Salvia } \\
\text { officinalis }\end{array}$} & $\begin{array}{l}\text { freeze- } \\
\text { drying }\end{array}$ & $\begin{array}{l}903.76 \pm \\
23.36 \mathrm{fg}, \mathrm{C}\end{array}$ & 38.72 & $\begin{array}{l}880.35 \pm \\
23.52^{\text {i, BC }}\end{array}$ & -2.59 & $\begin{array}{l}797.19 \pm \\
36.59 \mathrm{j}, \mathrm{B}\end{array}$ & -11.79 & $\begin{array}{l}696.57 \pm \\
34.02 \mathrm{~h}, \mathrm{~A}\end{array}$ & -22.92 \\
\hline & $\begin{array}{l}\text { natural } \\
\text { drying }\end{array}$ & $\begin{array}{l}925.10 \pm \\
68.11 \mathrm{~g}, \mathrm{~B}\end{array}$ & 42.00 & $\begin{array}{l}880.64 \pm \\
57.63 \text { i, B }\end{array}$ & -4.81 & $\begin{array}{l}837.41 \pm \\
10.49 \mathrm{k}, \mathrm{B}\end{array}$ & -9.48 & $\begin{array}{l}698.27 \pm \\
13.12^{\mathrm{h}, \mathrm{A}}\end{array}$ & -24.52 \\
\hline & $\begin{array}{l}\text { drying at } \\
30^{\circ} \mathrm{C}\end{array}$ & $\begin{array}{l}761.31 \pm \\
25.67^{\text {d, B }}\end{array}$ & 16.86 & $\begin{array}{l}728.49 \pm \\
42.77 \mathrm{~g}, \mathrm{~B}\end{array}$ & -4.31 & $\begin{array}{c}516.25 \pm \\
2.57^{\mathrm{f}, \mathrm{A}}\end{array}$ & -32.19 & $\begin{array}{l}491.67 \pm \\
33.68^{f, A}\end{array}$ & -35.42 \\
\hline & $\begin{array}{c}\text { drying at } \\
40^{\circ} \mathrm{C}\end{array}$ & $\begin{array}{l}684.65 \pm \\
20.86^{c, D}\end{array}$ & 5.09 & $\begin{array}{l}585.95 \pm \\
10.38 \text { ef, C }\end{array}$ & -14.42 & $\begin{array}{l}472.21 \pm \\
5.19 \text { de, B }\end{array}$ & -31.03 & $\begin{array}{l}379.18 \pm \\
10.65 \mathrm{~d}, \mathrm{~A}\end{array}$ & -44.62 \\
\hline & $\begin{array}{c}\text { drying at } \\
50^{\circ} \mathrm{C}\end{array}$ & $\begin{array}{l}502.64 \pm \\
28.70 \text { a, B }\end{array}$ & -22.85 & $\begin{array}{l}421.65 \pm \\
18.44 \mathrm{~b}, \mathrm{~A}\end{array}$ & -16.11 & $\begin{array}{c}425.38 \pm \\
18.44^{\mathrm{bc}, \mathrm{A}}\end{array}$ & -15.37 & $\begin{array}{l}382.54 \pm \\
21.07^{\mathrm{d}, \mathrm{A}}\end{array}$ & -23.89 \\
\hline \multirow{5}{*}{$\begin{array}{l}\text { Salvia } \\
\text { sclarea }\end{array}$} & $\begin{array}{l}\text { freeze- } \\
\text { drying }\end{array}$ & $\begin{array}{l}623.16 \pm \\
23.58 \mathrm{~b}, \mathrm{D}\end{array}$ & 9.62 & $\begin{array}{l}579.74 \pm \\
18.73 \text { ef, C }\end{array}$ & -6.97 & $\begin{array}{l}504.06 \pm \\
2.68 \text { ef, B }\end{array}$ & -19.11 & $\begin{array}{c}321.65 \pm \\
2.61^{\mathrm{c}, \mathrm{A}}\end{array}$ & -48.38 \\
\hline & $\begin{array}{l}\text { natural } \\
\text { drying }\end{array}$ & $\begin{array}{c}661.59 \pm \\
21.30^{b c, C}\end{array}$ & 16.38 & $\begin{array}{l}627.05 \pm \\
26.76^{\mathrm{f}, \mathrm{BC}}\end{array}$ & -5.22 & $\begin{array}{l}582.52 \pm \\
15.98 \mathrm{~g}, \mathrm{~B}\end{array}$ & -11.95 & $\begin{array}{l}364.53 \pm \\
7.87^{\mathrm{d}, \mathrm{A}}\end{array}$ & -44.90 \\
\hline & $\begin{array}{l}\text { drying at } \\
30^{\circ} \mathrm{C}\end{array}$ & $\begin{array}{l}606.23 \pm \\
29.43 \mathrm{~b}, \mathrm{C}\end{array}$ & 6.64 & $\begin{array}{l}552.12 \pm \\
21.29 \mathrm{de}, \mathrm{C}\end{array}$ & -8.93 & $\begin{array}{l}461.04 \pm \\
34.12^{\mathrm{cd}, \mathrm{B}}\end{array}$ & -23.95 & $\begin{array}{l}306.36 \pm \\
13.34^{c, ~ A}\end{array}$ & -49.46 \\
\hline & $\begin{array}{c}\text { drying at } \\
40^{\circ} \mathrm{C}\end{array}$ & $\begin{array}{l}544.32 \pm \\
18.56^{a}, D\end{array}$ & -4.25 & $\begin{array}{l}507.08 \pm \\
7.96^{\mathrm{cd}, \mathrm{C}}\end{array}$ & -6.84 & $\begin{array}{l}461.79 \pm \\
5.32 \mathrm{~cd}, \mathrm{~B}\end{array}$ & -15.16 & $\begin{array}{l}249.15 \pm \\
2.66^{\mathrm{b}, \mathrm{A}}\end{array}$ & -54.23 \\
\hline & $\begin{array}{l}\text { drying at } \\
50^{\circ} \mathrm{C}\end{array}$ & $\begin{array}{c}500.07 \pm \\
7.96^{\mathrm{a}}, \mathrm{C}\end{array}$ & -12.04 & $\begin{array}{c}459.19 \pm \\
10.50 \text { bc, BC }\end{array}$ & -8.18 & $\begin{array}{l}420.82 \pm \\
29.17^{b}, \mathrm{~B}\end{array}$ & -15.85 & $\begin{array}{l}227.92 \pm \\
15.67^{\mathrm{b}}, \mathrm{A}\end{array}$ & -54.42 \\
\hline
\end{tabular}

Results are expressed as mean $\pm \mathrm{SD}(n=3)$. DW-dry weight. Mean values with different letters (a-k) within the individual storage periods (columns) are statistically different $(p<0.05)$. Mean values with different letters (A-D) within the individual species of sage and drying methods (rows) are statistically different $(p<0.05)$. ${ }^{*}$ Changes in the antioxidant activity with reference to fresh sage samples. ${ }^{* *}$ Changes in the antioxidant activity with reference to sage samples directly after drying.

\section{Discussion}

As there are only very limited studies on the herb Salvia hispanica, the basic chemical composition, bioactive compounds, and antioxidant activity were analyzed and compared to other known species of sage (Salvia officinalis and Salvia sclarea). As drying is one of the most commonly used methods for herb preservation and shelf-life extension, the effects of different drying methods and periods of storage on the bioactive properties of the analyzed species of sage were evaluated.

In the tested samples, dietary fiber was the most abundant ingredient and fat was the least abundant. In the available literature, there are limited data regarding the basic chemical composition of sage. Peiretti and Gai [29], who studied Salvia hispanica, reported a lower content of ash $\left(7.7 \mathrm{~g} \cdot 100 \mathrm{~g} \mathrm{~g}^{-1} \mathrm{DW}\right)$ and protein $\left(5.7 \mathrm{~g} \cdot 100 \mathrm{~g}^{-1} \mathrm{DW}\right)$ compared to our results. Other results indicated that different herbs from Lamiaceae family were also characterized by a lower content of ash, protein, carbohydrates, and dietary fiber [35-37].

The content of total carotenoids in fresh Salvia officinalis was $63.48 \mathrm{mg} \cdot 100 \mathrm{~g}^{-1} \mathrm{DW}$. Daly et al. [38], Cvitković et al. [39], and Murkovic et al. [40], who studied commonly consumed herbs, reported a lower level of total carotenoids in sage. However, Martins et al. [41] showed higher values for Salvia officinalis leaves, compared to our results ob- 
tained for the whole herb (leaves and stems). This suggests that carotenoids are mainly accumulated in the leaves of the herb. This was confirmed also on the basis of research on different herbs [42]. To the best of our knowledge, the content of total carotenoids in Salvia hispanica and Salvia sclarea herb has not previously been determined.

Carotenoids are thermally and oxidatively labile compounds. They contain a chromophore group, which consists of seven or more conjugated double bonds. This structure allows the compounds to absorb visible light, giving them the appropriate color. This system is also responsible for the high thermal and oxidative sensitivity [43]. The obtained results showed that all the drying methods used caused a decrease in the carotenoids content in sage. The lowest loss was found after freeze-drying. During freeze-drying, a low temperature is used, and contact with oxygen is limited, which results in reduced degradation of these compounds [44]. On the other hand, the greatest losses of carotenoids were measured in samples after drying at $30^{\circ} \mathrm{C}$. This could be due to easier oxidation of these components at higher temperatures in comparison to freeze-drying, as well as longer contact with oxygen compared to drying at higher temperatures $\left(40{ }^{\circ} \mathrm{C}\right.$ and $\left.50{ }^{\circ} \mathrm{C}\right)$ [44]. Furthermore, thermal processes can damage some cellular structures, thereby releasing oxidative enzymes that decompose some antioxidant compounds such as carotenoids [45]. Generally, consumers store herbs at room temperature, and therefore the studied samples were stored under these conditions. A low temperature preserves quality, including the content of bioactive components [46]. On the other hand, storage at room temperature may cause loss of these compounds, as observed in our study. It is difficult to indicate the drying method that allows for the preservation of carotenoids during storage to the highest extent.

The content of total polyphenols in Salvia hispanica was $9.76 \mathrm{~g} \cdot 100 \mathrm{~g}^{-1} \mathrm{DW}$. To the best of our knowledge, there are no previous data on content of total polyphenolic compounds in the herb Salvia hispanica. Our results indicated that the herb Salvia officinalis contained $9.47 \mathrm{~g} \cdot 100 \mathrm{~g}^{-1} \mathrm{DW}$ of total polyphenols. However, Jeshvaghani et al. [47] showed that Salvia officinalis leaves had $8.64 \mathrm{mg} \cdot 100 \mathrm{~g}^{-1} \mathrm{DW}$ of polyphenolic compounds, while Roby et al. [48] reported a value of $0.595 \mathrm{~g} \cdot 100 \mathrm{~g}^{-1}$ DW. Dent et al. [49], who studied ethanol and water extracts of sage from Croatia, also obtained lower values. The herb Salvia officinalis from Bulgaria, studied by Atanassova et al. [50], had $27.94 \mathrm{mg} \cdot 100 \mathrm{~g}^{-1} \mathrm{DW}$ of polyphenolic compounds. The level of total polyphenols in Salvia sclarea was $4.95 \mathrm{~g} \cdot 100$ $\mathrm{g}^{-1}$ DW. Jeshvaghani et al. [47] obtained a higher value of $10.33 \mathrm{~g} \cdot 100 \mathrm{~g}^{-1} \mathrm{DW}$, while Taârit et al. [16] reported a lower level of polyphenols of $2.44 \mathrm{~g} \cdot 100 \mathrm{~g}^{-1} \mathrm{DW}$. Our results showed that the herb Salvia hispanica contained polyphenols at the same level as the herb Salvia officinalis. This suggests that the unknown Salvia hispanica herb, like the well-known Salvia officinalis, may have similar beneficial properties.

In all the tested species of sage, rosmarinic acid was the most abundant polyphenolic compound. The results of other studies also indicated that rosmarinic acid was the dominant polyphenolic compound in Salvia officinalis. Dent et al. [51] studied Salvia officinalis leaves from four wild field sites in the Mediterranean region of Croatia. Farhat et al. [52] analyzed Salvia officinalis from two different regions in north Tunisia at the vegetative, flowering, and fruiting stages. Hamrouni-Sellami et al. [53] evaluated Salvia officinalis from southern Tunisia, while Dent et al. [49] tested Salvia officinalis from the island of Pag (Croatia). These authors also detected phenolic acids such as p-hydroxybenzoic acid, caffeic acid, ferulic acid, gallic acid, p-coumaric acid, syringic acid, and vanillic acid, as well as flavonoids, mainly luteolin and apigenin (and their derivatives), hispidulin, and naringin. Additionally, Farhat et al. [52] and Hamrouni-Sellami et al. [53] identified the phenolic diterpenes carnosic acid and carnosol. Amato et al. [27], who for the first time showed a qualitative profile of the polyphenols in Salvia hispanica leaves, also detected compounds such as caffeic acid, chlorogenic acid, ferulic acid, rosmarinic acid, apigenin, luteolin, kaempferol, quercetin, and others. To the best of our knowledge, the polyphenol profile of the Salvia sclarea herb and the quantitative analysis of polyphenolic compounds in Salvia hispanica have not yet been evaluated. 
The obtained results indicated that Salvia hispanica and Salvia officinalis, after freezedrying, were characterized by the highest content of total polyphenols. Similar observations for Salvia officinalis were made by Sadowska et al. [31]. It is known that the low temperature of this process can protect polyphenolic compounds. Additionally, limited oxygen access can minimize the oxidation of these compounds [54]. Due to modification of the microstructure of freeze-dried plant tissues, the dried product is more porous, and thus the extraction process is more effective [55]. Furthermore, this process can inhibit the activity of some enzymes such as polyphenol oxidases, resulting in the protection of polyphenolic compounds [56]. Different polyphenol profiles in dried herbs, in comparison to fresh ones, can be due to the release of compounds after thermal treatment. The elevated temperature can influence the cell wall structure, promoting the release of internal compounds from the plant cells [57]. The greatest loss of polyphenols was found in samples of sage after drying at $50{ }^{\circ} \mathrm{C}$, caused by the use of the highest temperature. Moreover, during natural drying (in comparison to freeze-drying) the degradation of polyphenol oxidases was not immediate; therefore, oxidation of these compounds may occur during the process [56]. The obtained results do not allow an indication of the drying method that protects polyphenols during storage to the highest extent.

Our research indicated that Salvia hispanica and Salvia officinalis were characterized by higher antioxidant activity compared to Salvia sclarea. These results were in agreement with the data reported by Jeshvaghani et al. [47], who studied various species of sage. However, Stagos et al. [6] indicated that the herb Salvia sclarea had the highest antioxidant activity among all the tested sage samples. The results of our study showed that the herb Salvia hispanica was characterized by a fairly high antioxidant activity.

\section{Conclusions}

The obtained results showed that the herb of a barely investigated species of sage, Salvia hispanica, was rich in polyphenolic compounds and showed high antioxidant activity. Both the polyphenol content and the antioxidant capacity were on the same level as those determined in the well-known Salvia officinalis, and were significantly higher compared to those found in Salvia sclarea. In all tested species of sage, rosmarinic acid was the most abundant polyphenolic compound. Furthermore, Salvia hispanica was rich in sinapinic acid, naringin, rutin, and carnosol. The use of different drying methods allowed us to indicate freeze-drying as the most effective for preserving polyphenols and carotenoids in the studied species of sage. Long-term storage up to 12 months resulted in a gradual reduction in antioxidant activity as well as in the content of polyphenols and carotenoids. However, even after such a long period of storage the herbs of all tested species of sage showed strong antioxidant activity and a high content of bioactive compounds. To conclude, the herb Salvia hispanica showed a high potential to be used as a rich source of bioactive compounds, including antioxidants, in the food industry, cosmetics, biomaterials, and active food packaging, similarly to the well-known Salvia officinalis.

Author Contributions: Conceptualization, K.D.; methodology, K.D., A.K., and U.S.; investigation, K.D., M.D., and U.S.; data analysis, K.D., M.D., and K.C.-K.; writing-original draft preparation, K.D.; writing-review and editing, A.K., M.D., K.C.-K., and U.S.; supervision, A.K.; funding acquisition, K.C.-K. All authors have read and agreed to the published version of the manuscript.

Funding: This work was supported by the National Science Centre, Poland, grant no. 2017/27/B/ST8/ 00195 (KCK).

Institutional Review Board Statement: Not applicable.

Informed Consent Statement: Not applicable.

Data Availability Statement: The data presented in this study are available on request from the corresponding author.

Conflicts of Interest: The authors declare no conflict of interest. 
Sample Availability: Samples of the compounds are not available from the authors.

\section{References}

1. World Health Organization; Department of Technical Cooperation for Essential Drugs and Traditional. WHO Guidelines for Assessing Quality of Herbal Medicines with Reference to Contaminants and Residues. Medicine; WHO: Geneva, Switzerland, 2007.

2. Yun, J.W. Possible anti-obesity therapeutics from nature-A review. Phytochemistry 2010, 71, 1625-1641. [CrossRef] [PubMed]

3. Sergent, T.; Vanderstraeten, J.; Winand, J.; Beguin, P.; Schneider, Y.J. Phenolic compounds and plant extracts as potential natural anti-obesity substances. Food Chem. 2012, 135, 68-73. [CrossRef]

4. Walker, J.B.; Sytsma, K.J.; Treutlein, J.; Wink, M. Salvia (Lamiaceae) is not monophyletic: Implications for the systematics, radiation, and ecological specializations of Salvia and tribe Mentheae. Am. J. Bot. 2004, 91, 1115-1125. [CrossRef]

5. Russo, A.; Formisano, C.; Rigano, D.; Senatore, F.; Delfine, S.; Cardile, V.; Rosselli, S.; Bruno, M. Chemical composition and anticancer activity of essential oils of Mediterranean sage (Salvia officinalis L.) grown in different environmental conditions. Food Chem. Toxicol. 2013, 55, 42-47. [CrossRef] [PubMed]

6. Stagos, D.; Portesis, N.; Spanou, C.; Mossialos, D.; Aligiannis, N.; Chaita, E.; Panagoulis, C.; Reri, E.; Skaltsounis, L.; Tsatsakis, A.M.; et al. Correlation of total polyphenolic content with antioxidant and antibacterial activity of 24 extracts from Greek domestic Lamiaceae species. Food Chem. Toxicol. 2012, 50, 4115-4124. [CrossRef]

7. Douglas, T.E.L.; Kumari, S.; Dziadek, K.; Dziadek, M.; Abalymov, A.; Cools, P.; Brackman, G.; Coenye, T.; Morent, R.; Mohan, M.K.; et al. Titanium surface functionalization with coatings of chitosan and polyphenol-rich plant extracts. Mater. Lett. 2017, 196, 213-216. [CrossRef]

8. Dziadek, M.; Dziadek, K.; Checinska, K.; Zagrajczuk, B.; Golda-Cepa, M.; Brzychczy-Wloch, M.; Menaszek, E.; Kopec, A.; Cholewa-Kowalska, K. PCL and PCL/bioactive glass biomaterials as carriers for biologically active polyphenolic compounds: Comprehensive physicochemical and biological evaluation. Bioact. Mater. 2021, 6, 1811-1826. [CrossRef]

9. Dziadek, M.; Dziadek, K.; Zagrajczuk, B.; Menaszek, E.; Cholewa-Kowalska, K. Poly( $(\varepsilon$-caprolactone)/bioactive glass composites enriched with polyphenols extracted from sage (Salvia officinalis L.). Mater. Lett. 2016, 183, 386-390. [CrossRef]

10. Oudjedi, K.; Manso, S.; Nerin, C.; Hassissen, N.; Zaidi, F. New active antioxidant multilayer food packaging films containing Algerian Sage and Bay leaves extracts and their application for oxidative stability of fried potatoes. Food Control 2019, 98, 216-226. [CrossRef]

11. Baricevic, D.; Sosa, S.; Della Loggia, R.; Tubaro, A.; Simonovska, B.; Krasna, A.; Zupancic, A. Topical anti-inflammatory activity of Salvia officinalis L. leaves: The relevance of ursolic acid. J. Ethnopharmacol. 2001, 75, 125-132. [CrossRef]

12. Longaray Delamare, A.P.; Moschen-Pistorello, I.T.; Artico, L.; Atti-Serafini, L.; Echeverrigaray, S. Antibacterial activity of the essential oils of Salvia officinalis L. and Salvia triloba L. cultivated in South Brazil. Food Chem. 2007, 100, 603-608. [CrossRef]

13. Gniewosz, M.; Kraśniewska, K.; Wẹglarz, Z.; Przybył, J.L. The Comparison of Antimicrobial Properties of Ethanolic and Aqueous Extracts from Sage (Salvia officinalis L.). Bromatol. Chem. Toksykol. 2012, XLV, 743-748.

14. Kelen, M.; Tepe, B. Chemical composition, antioxidant and antimicrobial properties of the essential oils of three Salvia species from Turkish flora. Bioresour. Technol. 2008, 99, 4096-4104. [CrossRef] [PubMed]

15. Seol, G.H.; Shim, H.S.; Kim, P.J.; Moon, H.K.; Lee, K.H.; Shim, I.; Suh, S.H.; Min, S.S. Antidepressant-like effect of Salvia sclarea is explained by modulation of dopamine activities in rats. J. Ethnopharmacol. 2010, 130, 187-190. [CrossRef]

16. Taârit, M.B.; Msaada, K.; Hosni, K.; Marzouk, B. Fatty acids, phenolic changes and antioxidant activity of clary sage (Salvia sclarea L.) rosette leaves grown under saline conditions. Ind. Crop. Prod. 2012, 38, 58-63. [CrossRef]

17. Rajabi, Z.; Ebrahimi, M.; Farajpour, M.; Mirza, M.; Ramshini, H. Compositions and yield variation of essential oils among and within nine Salvia species from various areas of Iran. Ind. Crop. Prod. 2014, 61, 233-239. [CrossRef]

18. European Directorate for the Quality of Medicines \& HealthCare (EDQM). European Pharmacopoeia, 10th ed.; EDQM-European Directorate for the Quality of Medicines, Council of Europe: Strasbourg, France, 2011.

19. Hossain, M.B.; Barry-Ryan, C.; Martin-Diana, A.B.; Brunton, N.P. Effect of drying method on the antioxidant capacity of six Lamiaceae herbs. Food Chem. 2010, 123, 85-91. [CrossRef]

20. Mediani, A.; Abas, F.; Tan, C.P.; Khatib, A. Effects of different drying methods and storage time on free radical scavenging activity and total phenolic content of cosmos caudatus. Antioxidants 2014, 3, 358-370. [CrossRef]

21. Tiwari, U.; Cummins, E. Factors influencing levels of phytochemicals in selected fruit and vegetables during pre- and post-harvest food processing operations. Food Res. Int. 2013, 50, 497-506. [CrossRef]

22. Compaore, A.; Dissa, A.O.; Rogaume, Y.; Putranto, A.; Chen, X.D.; Mangindaan, D.; Zoulalian, A.; Rémond, R.; Tiendrebeogo, E. Application of the reaction engineering approach (REA) for modeling of the convective drying of onion. Dry. Technol. 2017, 35, 500-508. [CrossRef]

23. Hii, C.L.; Ong, S.P.; Yap, J.Y.; Putranto, A.; Mangindaan, D. Hybrid drying of food and bioproducts: A review. Dry. Technol. 2021, 39, 1554-1576. [CrossRef]

24. Ayerza, R.; Coates, W. Protein content, oil content and fatty acid profiles as potential criteria to determine the origin of commercially grown chia (Salvia hispanica L.). Ind. Crop. Prod. 2011, 34, 1366-1371. [CrossRef]

25. Marineli, R.d.S.; Moraes, É.A.; Lenquiste, S.A.; Godoy, A.T.; Eberlin, M.N.; Maróstica, M.R. Chemical characterization and antioxidant potential of Chilean chia seeds and oil (Salvia hispanica L.). LWT-Food Sci. Technol. 2014, 59, 1304-1310. [CrossRef] 
26. Sandoval-Oliveros, M.R.; Paredes-López, O. Isolation and characterization of proteins from chia seeds (Salvia hispanica L.). J. Agric. Food Chem. 2013, 61, 193-201. [CrossRef] [PubMed]

27. Amato, M.; Caruso, M.C.; Guzzo, F.; Galgano, F.; Commisso, M.; Bochicchio, R.; Labella, R.; Favati, F. Nutritional quality of seeds and leaf metabolites of Chia (Salvia hispanica L.) from Southern Italy. Eur. Food Res. Technol. 2015, 241, 615-625. [CrossRef]

28. da Silva Marineli, R.; Lenquiste, S.A.; Moraes, É.A.; Maróstica Jr, M.R. Antioxidant potential of dietary chia seed and oil (Salvia hispanica L.) in diet-induced obese rats. Food Res. Int. 2015, 76, 666-674. [CrossRef] [PubMed]

29. Peiretti, P.G.; Gai, F. Fatty acid and nutritive quality of chia (Salvia hispanica L.) seeds and plant during growth. Anim. Feed Sci. Technol. 2009, 148, 267-275. [CrossRef]

30. AOAC. Official Methods of Analysis, 18th ed.; Association of Offcial Analitical Chemists International: Gaintersburg, MD, USA, 2006.

31. Sadowska, U.; Kopeć, A.; Kourimska, L.; Zarubova, L.; Kloucek, P. The effect of drying methods on the concentration of compounds in sage and thyme. J. Food Process. Preserv. 2017, 41, e13286. [CrossRef]

32. Przetwory Owocowe i Warzywne-Przygotowanie Próbek i Metody Badań Fizykochemicznych—Oznaczanie Zawartości Sumy Karotenoidów i Beta-Karotenu. PN-A-75101-12. 1990. Available online: https://sklep.pkn.pl/pn-a-75101-03-1990p.html (accessed on 1 February 2022).

33. Dziadek, K.; Kopeć, A.; Piątkowska, E.; Leszczyńska, T.; Pisulewska, E.; Witkowicz, R.; Bystrowska, B.; Francik, R. Identification of polyphenolic compounds and determination of antioxidant activity in extracts and infusions of buckwheat leaves. Eur. Food Res. Technol. 2018, 244, 333-343. [CrossRef]

34. Dziadek, K.; Kopeć, A.; Czaplicki, S. The petioles and leaves of sweet cherry (Prunus avium L.) as a potential source of natural bioactive compounds. Eur. Food Res. Technol. 2018, 244, 1415-1426. [CrossRef]

35. Bandana, N.; Bala, N.; Verma, A. Utilization of locally available mint leaves concentrates for development of micronutrient rich food products. World J. Pharm. Pharm. Sci. 2015, 4, 1171-1177.

36. Sangwan, A.; Kawatra, A.; Sehgal, S. Nutrient composition of mint powder prepared from various drying methods. Nutr. Food Sci. 2012, 42, 21-25. [CrossRef]

37. Sulieman, A.M.E.; Abdelrahman, S.E.; Abdel Rahim, A.M. Phytochemical Analysis of Local Spearmint (Mentha spicata) Leaves and Detection of the Antimicrobial Activity of its Oil. J. Microbiol. Res. 2012, 1, 1-4. [CrossRef]

38. Daly, T.; Jiwan, M.A.; O'Brien, N.M.; Aherne, S.A. Carotenoid content of commonly consumed herbs and assessment of their Bioaccessibility using an in vitro digestion model. Plant Foods Hum. Nutr. 2010, 65, 164-169. [CrossRef] [PubMed]

39. Cvitkovi, D.; Repaji, M.; Pedisi, S. Composition and Antioxidant Properties of Pigments of Extraction Methods. Foods 2021, 10, 2477. [CrossRef] [PubMed]

40. Murkovic, M.; Gams, K.; Draxl, S.; Pfannhauser, W. Development of an Austrian carotenoid database. J. Food Compos. Anal. 2000, 13, 435-440. [CrossRef]

41. Martins, F.; Oliveira, I.; Barros, A.; Amaral, C.; Afonso, S.; Ferreira, H.; Gonçalves, B. Leaf age, seasonal and annual variations in Salvia officinalis L. Var. purpurascens biochemical characteristics. J. Appl. Bot. Food Qual. 2016, 89, 299-306.

42. Lisiewska, Z.; Kmiecik, W.; Korus, A. Content of vitamin C, carotenoids, chlorophylls and polyphenols in green parts of dill (Anethum graveolens L.) depending on plant height. J. Food Compos. Anal. 2006, 19, 134-140. [CrossRef]

43. Cardoso, W.S.; Borém, A.; Karam, D.; de Almeida Rios, S.; Paes, M.C.D. Influence of the moisture at harvest and drying process of the grains on the level of carotenoids in maize (Zea mays). Food Sci. Technol. 2015, 35, 481-486. [CrossRef]

44. Sojak, M.; Jaros, M.; Janaszek-Mańkowska, M.; Trajer, J.; Głowacki, S.; Ratajski, A. The effect of drying and long-term storage on colour and carotenoids content of giant pumpkin (Cucurbita maxima). Tech. Sci./Univ. Warm. Maz. Olsztyn 2016, 19, $295-312$.

45. Moreno G, D.C.; Díaz-Moreno, A.C. Efecto del proceso de secado por aire caliente en las características fisicoquímicas, antioxidantes y microestructurales de tomate cv. Chonto. Agron. Colomb. 2017, 35, 100-106.

46. Asgar, A. Effect of storage temperature and type of packaging on physical and chemical quality of carrot. IOP Conf. Ser. Earth Environ. Sci. 2020, 443, 012002. [CrossRef]

47. Jeshvaghani, Z.A.; Rahimmalek, M.; Talebi, M.; Goli, S.A.H. Comparison of total phenolic content and antioxidant activity in different Salvia species using three model systems. Ind. Crop. Prod. 2015, 77, 409-414. [CrossRef]

48. Roby, M.H.H.; Sarhan, M.A.; Selim, K.A.H.; Khalel, K.I. Evaluation of antioxidant activity, total phenols and phenolic compounds in thyme (Thymus vulgaris L.), sage (Salvia officinalis L.), and marjoram (Origanum majorana L.) extracts. Ind. Crop. Prod. 2013, 43, 827-831. [CrossRef]

49. Dent, M.; Dragović-Uzelac, V.; Penić, M.; Brñić, M.; Bosiljkov, T.; Levaj, B. The effect of extraction solvents, temperature and time on the composition and mass fraction of polyphenols in dalmatian wild sage (Salvia officinalis L.) extracts. Food Technol. Biotechnol. 2013, 51, 84-91.

50. Atanassova, M.; Georgieva, S.; Ivancheva, K. Total phenolic and total flavonoid contents, antioxidant capacity and biological contaminants in medicinal herbs. J. Univ. Chem. Technol. Metall. 2011, 46, 81-88.

51. Dent, M.; Kovačević, D.B.; Bosiljkov, T.; Dragović-Uzelac, V. Polyphenolic composition and antioxidant capacity of indigenous wild dalmatian sage (Salvia officinalis L.). Croat. Chem. Acta 2017, 90, 451-459. [CrossRef]

52. Ben Farhat, M.; Chaouch-Hamada, R.; Sotomayor, J.A.; Landoulsi, A.; Jordán, M.J. Antioxidant potential of Salvia officinalis L. residues as affected by the harvesting time. Ind. Crop. Prod. 2014, 54, 78-85. [CrossRef] 
53. Hamrouni-Sellami, I.; Rahali, F.Z.; Rebey, I.B.; Bourgou, S.; Limam, F.; Marzouk, B. Total Phenolics, Flavonoids, and Antioxidant Activity of Sage (Salvia officinalis L.) Plants as Affected by Different Drying Methods. Food Bioprocess Technol. 2013, 6, 806-817. [CrossRef]

54. Nawirska, A.; Figiel, A.; Kucharska, A.Z.; Sokół-Łetowska, A.; Biesiada, A. Drying kinetics and quality parameters of pumpkin slices dehydrated using different methods. J. Food Eng. 2009, 94, 14-20. [CrossRef]

55. Saifullah, M.; McCullum, R.; McCluskey, A.; Vuong, Q. Effects of different drying methods on extractable phenolic compounds and antioxidant properties from lemon myrtle dried leaves. Heliyon 2019, 5, e03044. [CrossRef] [PubMed]

56. Rababah, T.M.; Al-U’ Datt, M.; Alhamad, M.; Al-Mahasneh, M.; Ereifej, K.; Andrade, J.; Altarifi, B.; Almajwal, A.; Yang, W. Effects of drying process on total phenolics, antioxidant activity and flavonoid contents of common mediterranean herbs. Int. J. Agric. Biol. Eng. 2015, 8, 145-150.

57. Kayahan, S.; Saloglu, D. Comparison of Phenolic Compounds and Antioxidant Activities of Raw and Cooked Turkish Artichoke Cultivars. Front. Sustain. Food Syst. 2021, 5, 761145. [CrossRef] 